\title{
Higgs production in association with off-shell top-antitop pairs at NLO EW and QCD at the LHC
}

\author{
Ansgar Denner, ${ }^{a}$ Jean-Nicolas Lang, ${ }^{a}$ Mathieu Pellen ${ }^{a}$ and Sandro Uccirati ${ }^{b}$ \\ ${ }^{a}$ Universität Würzburg, Institut für Theoretische Physik und Astrophysik, \\ 97074 Würzburg, Germany \\ ${ }^{b}$ Università di Torino e INFN, \\ 10125 Torino, Italy \\ E-mail: ansgar.denner@physik.uni-wuerzburg.de, \\ jean-nicolas. lang@physik. uni-wuerzburg.de, \\ mathieu.pellen@physik.uni-wuerzburg.de, uccirati@to.infn.it
}

ABSTRACT: We present NLO electroweak corrections to Higgs production in association with off-shell top-antitop quark pairs. The full process $\mathrm{pp} \rightarrow \mathrm{e}^{+} \nu_{\mathrm{e}} \mu^{-} \bar{\nu}_{\mu} \mathrm{b} \overline{\mathrm{b} H}$ is considered, and hence all interference, off-shell, and non-resonant contributions are taken into account. The electroweak corrections turn out to be below one per cent for the integrated cross section but can exceed $10 \%$ in certain phase-space regions. In addition to its phenomenological relevance, the computation constitutes a major technical achievement as the full NLO virtual corrections involving up to 9-point functions have been computed exactly. The results of the full computation are supported by two calculations in the double-pole approximation. These also allow to infer the effect of off-shell contributions and emphasise their importance especially for the run II of the LHC. Finally, we present combined predictions featuring both NLO electroweak and QCD corrections in a common set-up that will help the experimental collaborations in their quest of precisely measuring the aforementioned process.

KEYWORDS: NLO Computations

ArXiv EPRINT: 1612.07138 


\section{Contents}

1 Introduction 1

2 Details of the calculation $\quad 2$

2.1 Real corrections 4

2.2 Virtual corrections 4

2.3 Double-pole approximation 6

$\begin{array}{lll}2.4 \text { Validation } & 7\end{array}$

3 Numerical results $\quad 8$

3.1 Input parameters and event selection 8

$\begin{array}{llr}3.2 & \text { EW corrections to integrated cross section } & 9\end{array}$

$\begin{array}{lll}3.3 & \text { EW corrections to differential distributions } & 11\end{array}$

$\begin{array}{lll}3.4 & \text { Comparison to the double-pole approximations } & 14\end{array}$

$\begin{array}{ll}\text { 3.5 Combination of NLO EW and QCD corrections } & 17\end{array}$

$\begin{array}{llr}4 \text { Conclusions } & 19\end{array}$

\section{Introduction}

Since the discovery of the Higgs boson [1, 2] at the Large Hadron Collider (LHC), significant experimental efforts have been devoted to probing its properties. Among these, the Higgs-boson couplings to other particles and in particular to the top quark are of prime importance. The measurement of Higgs-boson production in association with a pair of top quarks is a key input in that respect. The experimental measurement of this process is particularly challenging due to the large fraction of top quarks produced by other processes. Thus, so far only evidence for such a process has been achieved [3-7]. This measurement allows to examine possible new-physics contributions in the top-quark-Higgs Yukawa coupling. Hence, state-of-the-art predictions at next-to-leading-order (NLO) electroweak (EW) and QCD will soon be very valuable for the experimental collaborations in order to precisely measure the process and possibly discover new-physics mechanisms.

For the production of a Higgs boson in association with on-shell top quarks, already several NLO QCD computations have been performed [8-11], and their matching to parton showers [12-14] is also available. Moreover, resummation of soft-gluon-emission contributions for $\mathrm{t} \overline{\mathrm{t}} \mathrm{H}$ production has been performed to next-to-next-to-leading-logarithmic (NNLL) accuracy [15-18]. On the other hand, the computation of the NLO QCD corrections for off-shell top quarks has been realised only recently for the first time at the LHC [19] and at a linear collider [20]. Concerning NLO EW, only computations in the limit of on-shell top quarks [21-23] or in the narrow-width approximation [24] are available so far. With the 
present computation we go beyond the on-shell approximation and compute for the first time the full NLO EW corrections to Higgs-boson production in association with off-shell top quarks, i.e. the complete process pp $\rightarrow \mathrm{e}^{+} \nu_{\mathrm{e}} \mu^{-} \bar{\nu}_{\mu} \mathrm{b} \overline{\mathrm{b}} \mathrm{H}$. Hence, it features all nonresonant, interference, and off-shell effects allowing to make realistic predictions that can be directly compared to experiments. The present article follows a series of several NLO computations involving off-shell top quarks [19, 25, 26].

The presented computation is also a major technical achievement. While the Born and real contributions are of similar complexity as those in refs. [19, 25, 26], the virtual contributions are significantly more complicated. Indeed, this is the first time that an NLO computation involving one-loop amplitudes with up to 9-point functions is made public. So far, the most complicated NLO computations have been limited to 8-point functions [26$28]$. This progress is made possible by the use of the public codes ReCola $[29,30]$ and Collier [31, 32].

In addition to calculating the NLO EW corrections to the complete process, two computations in a double-pole approximation (DPA) are also provided. In the first one, two resonant $\mathrm{W}$ bosons are required while in the second, two resonant top quarks are demanded. On the one hand, the DPAs allow to estimate the size of non-resonant and off-shell contributions and hence to infer the validity of on-shell computations especially for differential distributions. Secondly, they constitute an important cross-check of the full computation. This is an important point as the full virtual amplitude is particularly involved. Both DPAs turn out to be very good approximations of the full computation.

Finally, in order to provide state-of-the-art predictions for Higgs-boson production in association with off-shell top quarks, we have recomputed the NLO QCD corrections presented in ref. [19]. In that way we can provide predictions featuring both NLO EW and QCD corrections to the full process in a consistent set-up. We present results for two different ways of combining NLO effects. The first one is an additive combination while the second one is a multiplicative combination.

In section 2 details concerning the calculation are provided. While sections 2.1 and 2.2 are devoted to the description of real and virtual corrections, respectively, in section 2.3 the two DPAs are reviewed, and in section 2.4 the validation of the computation is described. In section 3, numerical results are presented for a centre-of-mass energy of $\sqrt{s}=13 \mathrm{TeV}$ at the LHC. In particular, in section 3.1 the event selection and the input parameters are specified, and in sections 3.2 and 3.3 the results for the integrated cross sections and differential distributions are provided. The comparison between the full calculation and the two DPAs is performed in section 3.4 for both the total cross section and differential distributions. Finally, predictions for the LHC including both NLO EW and QCD are provided in section 3.5. Section 4 contains the conclusion.

\section{Details of the calculation}

In the present article, the NLO EW corrections to the full hadronic process

$$
\mathrm{pp} \rightarrow \mathrm{e}^{+} \nu_{\mathrm{e}} \mu^{-} \bar{\nu}_{\mu} \mathrm{b} \overline{\mathrm{b}} \mathrm{H}
$$



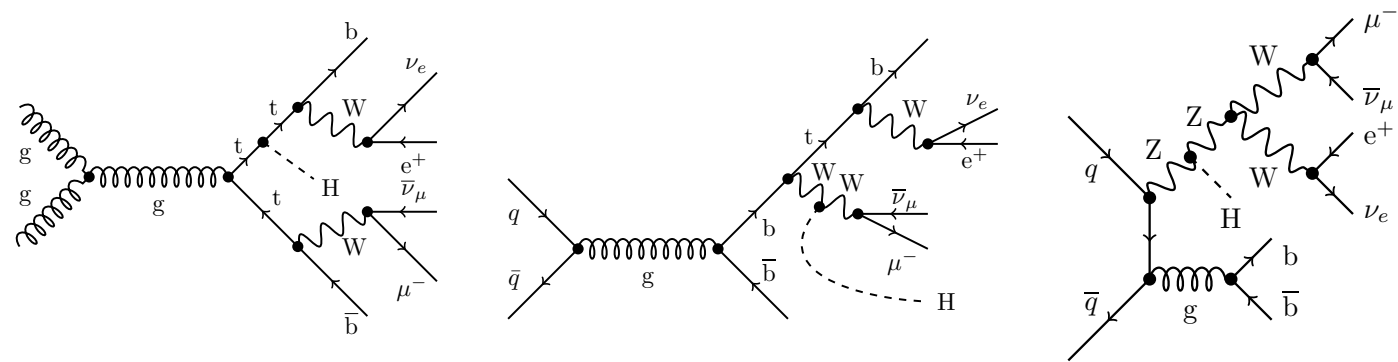

Figure 1. Sample tree-level Feynman diagrams with two (left), one (middle) and no (right) topquark resonances.
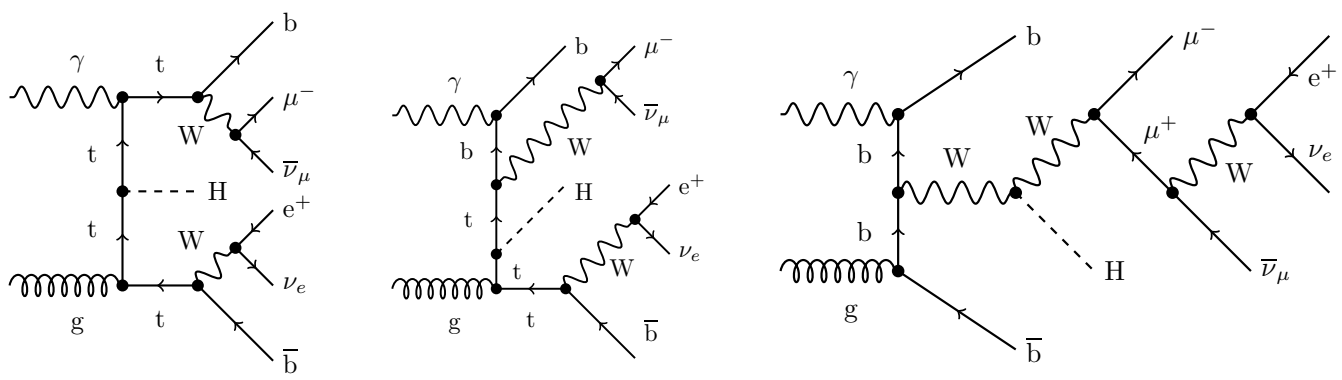

Figure 2. Sample tree-level Feynman diagrams with photons in the initial state.

are presented. We consider the lowest-order cross section of the order $\mathcal{O}\left(\alpha_{\mathrm{S}}^{2} \alpha^{5}\right)$. The EW corrections to this process consist of all possible corrections of the order $\mathcal{O}\left(\alpha_{\mathrm{s}}^{2} \alpha^{6}\right)$. We have neglected the LO electroweak process of order $\mathcal{O}\left(\alpha^{7}\right)$, while the LO interferences of order $\mathcal{O}\left(\alpha_{\mathrm{s}} \alpha^{6}\right)$ vanish. For reference, we have computed the Born process with initial state $\gamma \mathrm{g}$ of order $\mathcal{O}\left(\alpha_{\mathrm{s}} \alpha^{6}\right)$ and choose to present the corresponding results separately. We have not calculated the QCD corrections to this process, which are of order $\mathcal{O}\left(\alpha_{\mathrm{s}}^{2} \alpha^{6}\right)$, as the photon-induced process contributes only at the per-cent level and thus these corrections are negligible. The calculation presented here includes all interferences, resonant, non-resonant, and off-shell effects of the massive intermediate particles, i.e. the top quarks and the gauge bosons. In figure 1, representative LO diagrams featuring two, one, and no resonant top quark(s) are shown for gluon- and quark-induced processes. In figure 2, three diagrams of the photon-initiated process are depicted. Note that the quark-mixing matrix has been assumed to be diagonal, and the bottom-quark parton distribution function (PDF) has been neglected.

To perform the numerical integration, an in-house multi-channel Monte Carlo program MoCANLO [33] has been employed. It has already been used for several computations involving processes with high multiplicity [19, 26, 28]. The multi-channel phase-space integration follows the ones of refs. [34-36]. The infrared (IR) singularities in the real contributions are treated with the dipole subtraction method [37-39]. All matrix-elements 

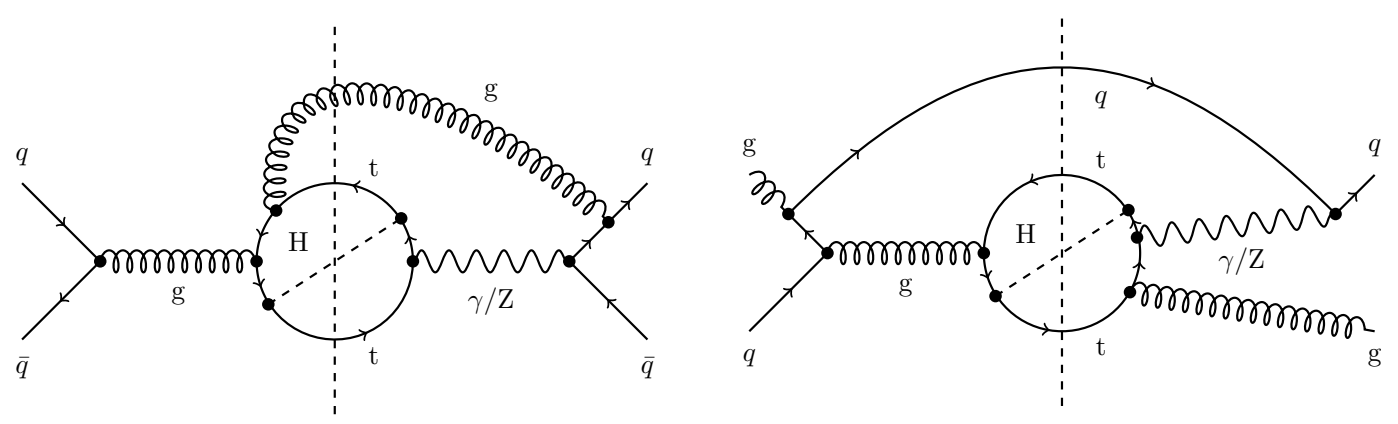

Figure 3. Two Feynman diagrams squared for real corrections featuring interference between QCD and EW tree-level diagrams. The decay products of the top quarks are not shown as their inclusion does not alter the discussion.

are provided by the computer code RECOLA-1.1 $[29,30]^{1}$ and the loop-integral library Collier-1.1 [31, 32]..$^{2}$

\section{$2.1 \quad$ Real corrections}

The real corrections to the process (2.1) consist of all real-radiation contributions at the order $\mathcal{O}\left(\alpha_{\mathrm{s}}^{2} \alpha^{6}\right)$. The first type of corrections results from photons radiated off any of the charged particles. In addition, interferences of a QCD-mediated process emitting a gluon with its EW counterpart in the $q \bar{q}$ channel must be taken into account. This is exemplified on the left-hand side of figure 3 where for simplicity on-shell top quarks are represented even if the computation comprises off-shell top quarks. Finally, a last type of interference appears in the $q \mathrm{~g}$ or $\bar{q} \mathrm{~g}$ channel as shown on the right-hand side of figure 3 .

The Catani-Seymour subtraction formalism for QCD [37, 38] and QED [39] has been used for the treatment of the IR singularities. Both the QCD and QED singularities from collinear initial-state splittings have been absorbed in the PDFs using the $\overline{\mathrm{MS}}$ factorisation scheme. We use the LUXqed_plus_PDF4LHC15_nnlo_100 set [40] that provides a $\overline{\mathrm{MS}}$ photon PDF. Note that all ingredients needed for the real-subtracted part (squared amplitudes for the real-correction processes as well as the colour- and spin-correlated squared amplitudes) have been obtained from the computer code RECOLA [29, 30].

\subsection{Virtual corrections}

Analogously to the real corrections, two types of virtual corrections must be taken into account. The first type arises from one-loop amplitudes of order $\mathcal{O}\left(\alpha_{\mathrm{S}} \alpha^{7 / 2}\right)$ interfered with tree amplitudes of order $\mathcal{O}\left(\alpha_{\mathrm{S}} \alpha^{5 / 2}\right)$. For the $\bar{q} q$ channels, the second type of corrections results from the interference of one-loop amplitudes of order $\mathcal{O}\left(\alpha_{\mathrm{S}}^{2} \alpha^{5 / 2}\right)$ with tree amplitudes of order $\mathcal{O}\left(\alpha^{7 / 2}\right)$. A sketch of these two types of corrections is shown in figure 4 . As for the interferences in the real corrections, the decay products of the top quarks are not

\footnotetext{
${ }^{1}$ It is publicly available at https://recola.hepforge.org.

${ }^{2}$ It is publicly available at https://collier.hepforge.org.
} 

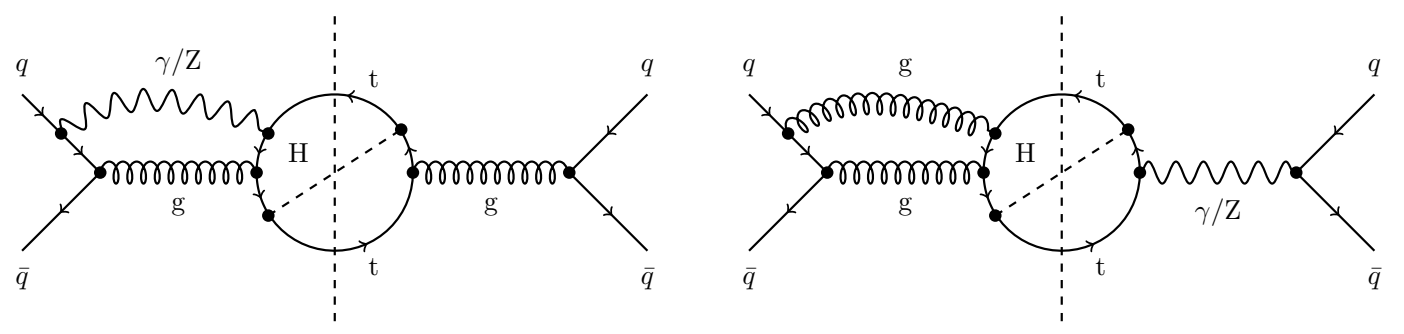

Figure 4. Sample one-loop Feynman diagrams squared. On the left-hand side, the diagram represents an EW correction to the QCD process which can also be seen as a QCD correction to the EW amplitude interfered with the QCD amplitude. On the right-hand side, a QCD correction to the QCD amplitude interfered with the EW amplitude is displayed. The decay products of the top quarks are not shown as their inclusion does not alter the discussion.
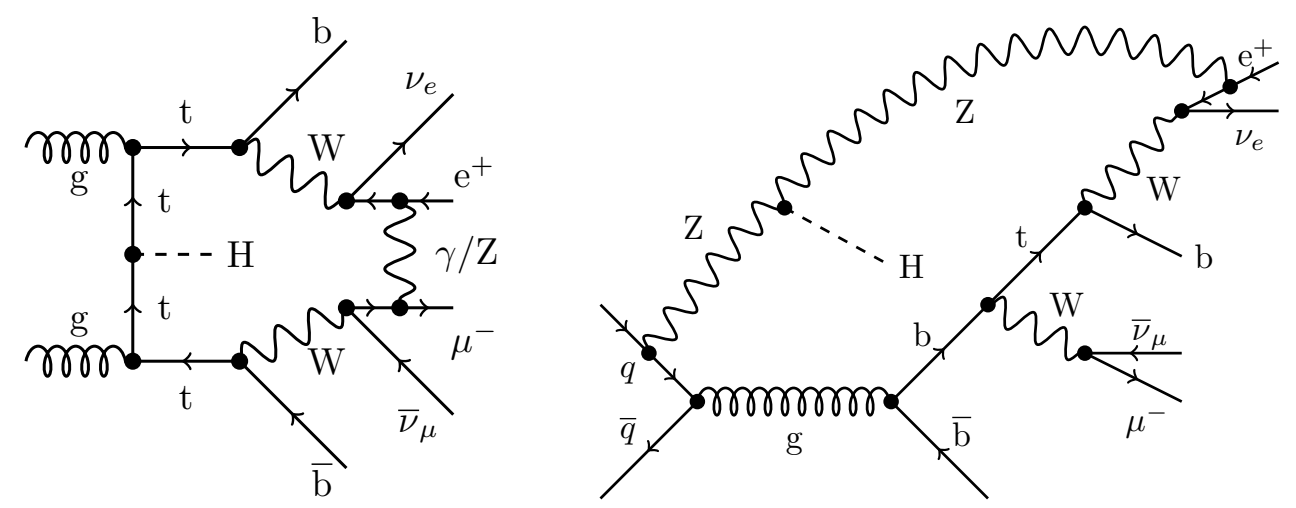

Figure 5. Example of nonagon and octagon one-loop Feynman diagrams.

shown since their inclusion does not alter the discussion. Two of the most complicated loop diagrams (8- and 9-point functions) are displayed in figure 5. These virtual contributions are provided by the matrix-element generator RECOLA [29, 30] in the 't Hooft-Feynman gauge in dimensional regularisation. The Collien library $[31,32]$ is used to calculate the one-loop scalar [41-44] and tensor integrals [45-47] numerically. Note that this is the first time that a NLO computation featuring 9-point functions has been made public. As discussed in section 2.4, the 9-point functions yield sizeable contributions to the full process. This demonstrates the ability of the computer codes RECOLA and COLLIER to provide fast and reliable one-loop amplitudes for complicated processes.

All resonant massive particles (top quarks, Z bosons and $\mathrm{W}$ bosons) are treated in the complex-mass scheme [35, 48, 49], where the masses of the unstable particles as well as the weak mixing angle are complex quantities,

$$
\bar{M}_{\mathrm{W}}^{2}=M_{\mathrm{W}}^{2}-\mathrm{i} M_{\mathrm{W}} \Gamma_{\mathrm{W}}, \quad \bar{M}_{\mathrm{Z}}^{2}=M_{\mathrm{Z}}^{2}-\mathrm{i} M_{\mathrm{Z}} \Gamma_{\mathrm{Z}}, \quad \text { and } \quad \cos \theta_{\mathrm{w}}=\frac{\bar{M}_{\mathrm{W}}}{\bar{M}_{\mathrm{Z}}}
$$




\subsection{Double-pole approximation}

In ref. [26], two DPAs have been presented for the process $\mathrm{pp} \rightarrow \mathrm{e}^{+} \nu_{\mathrm{e}} \mu^{-} \bar{\nu}_{\mu} \mathrm{b} \overline{\mathrm{b}}$ at NLO EW. As the Higgs boson is neither electrically charged nor charged under QCD, all formulas presented in ref. [26] can be applied to $\mathrm{pp} \rightarrow \mathrm{e}^{+} \nu_{\mathrm{e}} \mu^{-} \bar{\nu}_{\mu} \mathrm{b} \overline{\mathrm{b}} \mathrm{H}$ straightforwardly. Therefore, we do not provide a detailed description here, and the interested reader is referred to ref. [26] and references therein.

The DPA serves two key purposes. First, since it selects resonant contributions, one can infer the effect of non-resonant and off-shell contributions upon comparing with the full calculation. This, in particular, reveals if on-shell approximations can approximate satisfactorily the full process. Second, since the DPA turns out to be a very good approximation to the full calculation (see below), it serves as a check of the full virtual corrections.

As opposed to on-shell approximations, the DPA accounts for some off-shell effects. Indeed, the resonant propagator and the phase space are exactly taken into account, while the rest of the matrix element is expanded about the resonance poles. Finally, the DPA also accounts for resonant non-factorisable corrections. Following refs. [50, 51], the pole approximation has been applied only to the virtual corrections. Thus, in the LO and all real contributions no pole approximation is utilised. But in order to cancel the IR singularities originating from the virtual corrections, one has to apply the on-shell projection to the terms containing the $I$-operator in the integrated dipole contribution. ${ }^{3}$ On the other hand, the $P$ and $K$-operator terms are still evaluated with the off-shell kinematics like the real corrections. This introduces a mismatch, which is of the order of the intrinsic error of the DPA.

The process pp $\rightarrow \mathrm{e}^{+} \nu_{\mathrm{e}} \mu^{-} \bar{\nu}_{\mu} \mathrm{b} \overline{\mathrm{b}} \mathrm{H}$ is dominated by the production of two top quarks that decay into bottom quarks and lepton-neutrino pairs via $\mathrm{W}$ bosons. Requiring either two resonant top quarks or two resonant $\mathrm{W}$ bosons is thus expected to approximate well the full process. We have studied two different DPAs for the process (2.1): in one case (WW DPA), two resonant $\mathrm{W}$ bosons are demanded, while in the second case (tt DPA), two resonant top quarks are required. The momenta of the resonant particles entering the matrix elements have to be projected on shell in order to ensure gauge invariance. The two on-shell projections are identical to the ones of ref. [26]. For the WW DPA, the on-shell projection is designed such that the invariants of the top quarks are left untouched. Analogously, for the on-shell projection of tt DPA, the invariants of the $\mathrm{W}$ bosons are not modified.

In the DPA, two different kinds of corrections appear, factorisable and non-factorisable corrections. The factorisable virtual corrections can be uniquely attributed either to the production of the resonant particles or to their decays. For a pole approximation (PA) with $r$ resonances ( $r=2$ for a DPA), they can be written as [52]

$$
\begin{aligned}
\mathcal{M}_{\text {virt,fact,PA }}=\sum_{\lambda_{1}, \ldots, \lambda_{r}}\left(\prod_{i=1}^{r} \frac{1}{K_{i}}\right) & {\left[\mathcal{M}_{\mathrm{virt}}^{I \rightarrow N, \bar{R}} \prod_{j=1}^{r} \mathcal{M}_{\mathrm{LO}}^{j \rightarrow R_{j}}\right.} \\
& \left.+\mathcal{M}_{\mathrm{LO}}^{I \rightarrow N, \bar{R}} \sum_{k=1}^{r} \mathcal{M}_{\mathrm{virt}}^{k \rightarrow R_{k}} \prod_{j \neq k}^{r} \mathcal{M}_{\mathrm{LO}}^{j \rightarrow R_{j}}\right]_{\left\{\bar{k}_{l}^{2} \rightarrow \widehat{\bar{k}}_{l}^{2}=M_{l}^{2}\right\}_{l \in \bar{R}}} .
\end{aligned}
$$

${ }^{3}$ The $I-, P-$ and $K$-operator are defined in ref. [37]. 
The propagator of the resonant particle $i$ is $K_{i}=\bar{k}_{i}^{2}-\bar{M}_{i}^{2}$, where $\bar{M}_{i}^{2}=M_{i}^{2}-\mathrm{i} M_{i} \Gamma_{i}$ is its complex mass squared. The on-shell projection is applied everywhere in the matrix element but in the resonant propagators $K_{i}$ and is indicated by $\left\{\bar{k}_{l}^{2} \rightarrow \widehat{\bar{k}}_{l}^{2}=M_{l}^{2}\right\}$. The indices $I$, $\bar{R}, R_{i}$ and $N$ denote the ensembles of initial particles, resonant particles, decay products of the resonant particle $i$, and the final-state particles not resulting from the decay of a resonant particle. Finally $\lambda_{i}$ represents the polarisations of the resonance $i$. Note that these virtual factorisable corrections have been obtained via the computer code RECOLA, which allows to select resonant contributions at both LO and NLO.

When taking the on-shell limit of the momenta of the resonant particles, artificial IR singularities related to charged resonances are introduced. These artificial IR divergences are cancelled when including non-factorisable corrections. The non-factorisable corrections result from diagrams that do not split into production and decay parts by cutting only the resonant lines but also include contributions from factorisable diagrams [50]. The latter are obtained by taking the factorisable diagrams, where the IR singularities related to the resonant particles are regularised by the finite decay widths, and subtracting these contributions for zero decay width, which contains the artificial IR-divergent piece mentioned previously. The non-factorisable corrections can be written in the form

$$
2 \operatorname{Re}\left\{\mathcal{M}_{\mathrm{LO}, \mathrm{PA}}^{*} \mathcal{M}_{\text {virt,nfact,PA }}\right\}=\left|\mathcal{M}_{\mathrm{LO}, \mathrm{PA}}\right|^{2} \delta_{\text {nfact }},
$$

where $\delta_{\text {nfact }}$ is a function of scalar integrals (computed using the ColLIER library) whose expression can be found in refs. [26, 52].

As explained previously, the $q \bar{q}$ channels have two kinds of virtual NLO contributions connected by IR divergences: one-loop amplitudes of order $\mathcal{O}\left(\alpha_{\mathrm{s}} \alpha^{7 / 2}\right)$ interfered with tree amplitudes of order $\mathcal{O}\left(\alpha_{\mathrm{S}} \alpha^{5 / 2}\right)$ and the interference of one-loop amplitudes of order $\mathcal{O}\left(\alpha_{\mathrm{S}}^{2} \alpha^{5 / 2}\right)$ with tree amplitudes of order $\mathcal{O}\left(\alpha^{7 / 2}\right)$. Thus, the DPA must be applied to both types of virtual corrections.

\subsection{Validation}

The hadronic cross section for the full LO process as well as the photon-induced channels for dynamical scale have been checked against the computer code MADGraph5_AMC@NLO [53]. Agreement within the statistical error has been obtained. At the squared matrix-element level, we have checked all the tree-level contributions against MADGRAPH5_AMC@NLO. For Born, computing 10000 random phase-space points, we have found an agreement of at least 11 digits for $99 \%$ of the points, whereas for the real contribution, the two codes agree for $99 \%$ of the points within at least 8 digits.

To verify the IR and ultra-violet (UV) finiteness, the cross section has been computed for different IR and UV regulators, respectively. In order to check the proper subtraction of IR divergences the $\alpha$ parameter ${ }^{4}$ has been varied from $10^{-2}$ to 1 . The parameter $\alpha$ can restrict the phase space for the dipole subtraction terms to the vicinity of the singular regions [54] and thus improves numerical stability. We have also checked a

\footnotetext{
${ }^{4}$ The results presented in this article are obtained for $\alpha=10^{-2}$.
} 
Ward identity for the gluon-induced virtual amplitude, substituting in the one-loop amplitude the polarisation vector of one of the initial-state gluons by its momentum normalised to its energy, as $\epsilon_{\mathrm{g}}^{\mu} \rightarrow p_{\mathrm{g}}^{\mu} / p_{\mathrm{g}}^{0}$. Looking at the cumulative fraction of events with $\operatorname{Re}\left[\mathcal{M}_{0}^{*}\left(\epsilon_{\mathrm{g}}\right) \mathcal{M}_{1}\left(\epsilon_{\mathrm{g}} \rightarrow p_{\mathrm{g}} / p_{\mathrm{g}}^{0}\right)\right] / \operatorname{Re}\left[\mathcal{M}_{0}^{*}\left(\epsilon_{\mathrm{g}}\right) \mathcal{M}_{1}\left(\epsilon_{\mathrm{g}}\right)\right]$ larger than given values, we have found comparably good results to those of refs. $[19,26]$. We remark that the Ward identity is completely spoiled if we omit the contributions of 9-point functions. For the gluon-induced channel, the full virtual contribution to the integrated cross section is 0.0555(6). Without 9-point functions, it is $0.381(4)$, while the contribution of 9-point functions alone is $-0.322(5)$. This indicates that the 9-point functions yield a sizeable contributions to the cross section. Finally, to check the virtual corrections, two DPAs have been used and agree very well with the full computation (see below).

Concerning the QCD corrections, we have simply reproduced representative contributions of ref. [19] as this computation has undergone already numerous checks. Since we use the same Monte Carlo program and have access to the splitting of every single contribution, we have been able to check each of them in detail.

\section{Numerical results}

\subsection{Input parameters and event selection}

In this section, we present predictions for the LHC operating at a centre-of-mass energy $\sqrt{s}=13 \mathrm{TeV}$. In particular, the integrated cross section and differential distributions including NLO EW corrections are reported. The PDFs have been incorporated through LHAPDF 6.1.5 [55, 56]. Specifically, the LUXqed_plus_PDF4LHC15_nnlo_100 set [40] has been used for all LO and NLO results. It is next-to-next-to-leading-order (NNLO) accurate in QCD and includes all terms of order $\alpha L\left(\alpha_{s} L\right)^{n}, \alpha\left(\alpha_{s} L\right)^{n}$, and $\alpha^{2} L^{2}\left(\alpha_{s} L\right)^{n}$, where $L=\ln \left(\mu^{2} / m_{\mathrm{p}}^{2}\right), \mu$ is the renormalisation scale, and $m_{\mathrm{p}}$ is the proton mass. For the QCD partons, this PDF set is based on refs. [57-62]. Moreover, it features the inclusion of an $\overline{\mathrm{MS}}$ photon PDF needed for the photon-initiated contributions. For the renormalisation and factorisation scale, the following dynamical scale has been chosen [19]:

$$
\mu_{\mathrm{dyn}}=\left(m_{\mathrm{T}, \mathrm{t}} m_{\mathrm{T}, \overline{\mathrm{t}}} m_{\mathrm{T}, \mathrm{H}}\right)^{\frac{1}{3}} \quad \text { with } \quad m_{\mathrm{T}}=\sqrt{m^{2}+p_{\mathrm{T}}^{2}} .
$$

Note that contributions for bottom-quark PDFs have been neglected.

The $G_{\mu}$ scheme [50] has been used where $\alpha$ is obtained from the Fermi constant,

$$
\alpha=\frac{\sqrt{2}}{\pi} G_{\mu} M_{\mathrm{W}}^{2}\left(1-\frac{M_{\mathrm{W}}^{2}}{M_{\mathrm{Z}}^{2}}\right) \quad \text { with } \quad G_{\mu}=1.16637 \times 10^{-5} \mathrm{GeV}^{-2},
$$

leading to $\alpha=0.0075553105 \ldots$.

The numerical values for the masses and widths used in this computation read [63]:

$$
\begin{aligned}
m_{\mathrm{t}} & =173.34 \mathrm{GeV}, & \Gamma_{\mathrm{t}}^{\mathrm{NLO}} & =1.36918 \ldots \mathrm{GeV}, \\
M_{\mathrm{Z}}^{\mathrm{OS}} & =91.1876 \mathrm{GeV}, & \Gamma_{\mathrm{Z}}^{\mathrm{OS}} & =2.4952 \mathrm{GeV}, \\
M_{\mathrm{W}}^{\mathrm{OS}} & =80.385 \mathrm{GeV}, & & \Gamma_{\mathrm{W}}^{\mathrm{OS}}=2.085 \mathrm{GeV}, \\
M_{\mathrm{H}} & =125.0 \mathrm{GeV} . & &
\end{aligned}
$$


The masses and widths of all other quarks and leptons have been neglected. The effect of a finite bottom-quark mass on the cross section has been found to be below the per-cent level in our set-up [19]. The values of the top-quark widths (LO and NLO) have been taken from ref. [64], where both EW and QCD NLO corrections for massive bottom quarks have been calculated. The effect of the bottom-quark mass on the top-quark width has been found to be negligible compared to the integration errors on the cross section [26]. Note that the Higgs mass stated above is not the one used in ref. [64] but the one recommended by the Higgs Cross section Working Group [65] (such a change has a negligible impact on the NLO EW corrections). Finally, in this article we have used two different top-quark widths for the LO contributions. When presenting results for the NLO EW corrections (and their comparison to the two DPAs) the width $\Gamma_{\mathrm{t}}^{\mathrm{NLO}, \mathrm{QCD}}=1.35029 \mathrm{GeV}$ which features NLO QCD corrections is used at LO. This yields relative EW correction that can be directly compared to EW corrections in computations with on-shell top quarks. On the other hand, when presenting the combined NLO EW and QCD results, the width used for the LO predictions, $\Gamma_{\mathrm{t}}^{\mathrm{LO}}=1.449582 \mathrm{GeV}$, does not incorporate any NLO corrections.

The pole values for the gauge-boson $(V=\mathrm{W}, \mathrm{Z})$ parameters are obtained from the measured on-shell (OS) values of the masses and widths, according to ref. [66],

$$
M_{V}=M_{V}^{\mathrm{OS}} / \sqrt{1+\left(\Gamma_{V}^{\mathrm{OS}} / M_{V}^{\mathrm{OS}}\right)^{2}}, \quad \Gamma_{V}=\Gamma_{V}^{\mathrm{OS}} / \sqrt{1+\left(\Gamma_{V}^{\mathrm{OS}} / M_{V}^{\mathrm{OS}}\right)^{2}} .
$$

The anti- $k_{\mathrm{T}}$ algorithm [67], is used to cluster QCD partons and photons into jets as well as photons with light charged particles, using a jet-resolution parameter $R=0.4$. In the rapidity-azimuthal-angle plane, the distance between two particles $i$ and $j$ is defined as

$$
R_{i j}=\sqrt{\left(\Delta \phi_{i j}\right)^{2}+\left(y_{i}-y_{j}\right)^{2}}
$$

where $\Delta \phi_{i j}$ is the azimuthal-angle difference. The rapidity of jet $i$ reads $y_{i}=\frac{1}{2} \ln \frac{E+p_{z}}{E-p_{z}}$, where $E$ is the energy of the jet and $p_{z}$ the component of its momentum along the beam axis. Only final-state quarks, gluons, and charged fermions with rapidity $|y|<5$ are clustered into IR-safe objects.

After recombination, standard event selections are applied on the transverse momenta and rapidities of charged leptons and $\mathrm{b}$ jets, missing transverse momentum and rapidityazimuthal-angle distance between bottom jets. The Higgs boson is not included in the event selection. In the final state, two bottom jets and two charged leptons are required, and the following event selection is applied:

$$
\begin{array}{rrrr}
\text { bottom jets: } & p_{\mathrm{T}, \mathrm{b}}>25 \mathrm{GeV}, & \left|y_{\mathrm{b}}\right|<2.5, \\
\text { charged lepton: } & p_{\mathrm{T}, \ell}>20 \mathrm{GeV}, & \left|y_{\ell}\right|<2.5, \\
\text { missing transverse momentum: } & p_{\mathrm{T}, \mathrm{miss}}>20 \mathrm{GeV}, & \\
\text { bottom-jet-bottom-jet distance: } & \Delta R_{\mathrm{bb}}>0.4 . &
\end{array}
$$

\subsection{EW corrections to integrated cross section}

In this section the results for the integrated cross section for the LHC at a centre-ofmass energy of $\sqrt{s}=13 \mathrm{TeV}$ are presented. The different contributions are summarised 


\begin{tabular}{|cccc|}
\hline Ch. & $\sigma_{\mathrm{LO}}[\mathrm{fb}]$ & $\sigma_{\mathrm{NLO}} \mathrm{EW}[\mathrm{fb}]$ & $\delta[\%]$ \\
\hline $\mathrm{gg}$ & $2.0116(1)$ & $2.020(1)$ & +0.42 \\
$q \bar{q}$ & $0.84860(5)$ & $0.8454(6)$ & -0.38 \\
$\mathrm{~g} q(/ \bar{q})$ & \multicolumn{3}{c|}{$0.00007(2)$} \\
\hline$\gamma \mathrm{g}$ & \multicolumn{3}{c|}{$0.02178(1)$} \\
\hline $\mathrm{pp}$ & $2.8602(1)$ & $2.866(1)$ & +0.20 \\
\hline
\end{tabular}

Table 1. Contributions to the integrated cross section for $\mathrm{pp} \rightarrow \mathrm{e}^{+} \nu_{\mathrm{e}} \mu^{-} \bar{\nu}_{\mu} \mathrm{b} \overline{\mathrm{b}} \mathrm{H}(\gamma / \mathrm{j})$ at the LHC at a centre-of-mass energy of $\sqrt{s}=13 \mathrm{TeV}$. The quark-antiquark contributions include $q=\mathrm{u}, \mathrm{d}, \mathrm{c}, \mathrm{s}$. The contribution originating from the real radiation of a quark or an antiquark is denoted by $g q(/ \bar{q})$. The total cross section (denoted by pp) does not include the photon-induced channel (denoted by $\gamma \mathrm{g})$. The definition of the relative corrections reads $\delta=\sigma_{\mathrm{NLO}} \mathrm{EW} / \sigma_{\mathrm{LO}}$. The integration errors of the last digits are given in parentheses.

in table 1. These predictions are made for the input parameters given in eqs. (3.1)-(3.3) and the event selections defined in eq. (3.6). We consider LO contributions of the order $\mathcal{O}\left(\alpha_{\mathrm{s}}^{2} \alpha^{5}\right)$, while the EW NLO corrections arise at order $\mathcal{O}\left(\alpha_{\mathrm{s}}^{2} \alpha^{6}\right)$. The $\gamma \mathrm{g}$ contributions being of the order $\mathcal{O}\left(\alpha_{\mathrm{s}} \alpha^{5}\right)$, have not been included in the total cross section and are shown only for reference.

Due to the enhanced gluon PDF, the gluon-gluon-initiated channel is dominating. The $q \bar{q}$ channels including $q=\mathrm{u}, \mathrm{d}, \mathrm{c}, \mathrm{s}$ make up $30 \%$ of the total cross section. This is in contrast to the production of a pair of off-shell top quarks [26] where the $q \bar{q}$ channels account for only $12 \%$ of the total cross section. While the corrections to the gluon-gluon-initiated channel are positive, those to the $q \bar{q}$ channels are negative amounting $+0.42 \%$ and $-0.38 \%$, respectively. Moreover, the $g q / \bar{q}$ channels give a negligible contribution to the total cross section. In the end, for the full hadronic process, the EW corrections contribute $0.20 \%$. Note that the photon contributions account for $0.76 \%$ of the NLO cross section.

In ref. [22], EW corrections for the production of a Higgs boson in association with on-shell top quarks have been reported. In the $G_{\mu}$ scheme, they amount to $+1.8 \%$ and comprise photon contributions of $0.2 \%$. In our calculation the photon-induced contributions have not been included in the definition of the NLO EW corrections. Accounting for this effect, the two computations agree within $1.5 \%$. Note that cuts on the final state are applied in our computation which also includes off-shell and non-resonant contributions. By definition, these are not taken into account in on-shell computations.

Following ref. [19], the bottom quarks have been considered massless and the bottomquark PDF has been neglected. This is justified by the fact that they contribute at the per-mille level.

To conclude, the EW corrections are below the per-cent level for the integrated cross section. For differential distributions, on the other hand, the EW corrections have a larger impact (see below). 


\subsection{EW corrections to differential distributions}

Turning to differential distributions, two plots are shown for each observable. The upper panels display the LO and NLO EW predictions, while in the lower panels the relative corrections $\delta=\sigma_{\mathrm{NLO}} \mathrm{EW} / \sigma_{\mathrm{LO}}-1$ are shown in per cent. For reference, the $\gamma \mathrm{g}$ contribution is also displayed as $\delta_{\gamma \mathrm{g}}=\sigma_{\gamma \mathrm{g}} / \sigma_{\mathrm{LO}}$ and labelled photon. As opposed to the distributions shown in ref. [26], we have restricted the range of the transverse-momentum distributions to $400 \mathrm{GeV}$. As the process is just about to be measured, it is unlikely that the experimental collaborations will be able to probe the very tail of the distributions in a near future.

In figure 6 , a selection of transverse-momentum distributions is shown. In all distributions, the effects of the Sudakov logarithms at high transverse momenta are clearly visible. In general, the corrections vary between $1 \%$ and $4 \%$ for transverse momenta below $50 \mathrm{GeV}$ and grow negative towards high transverse momenta. At the end of the range shown $(400 \mathrm{GeV})$, the EW corrections reach up to $-8 \%$.

This is exemplified in figure $6 \mathrm{a}$, where the distribution of the muon transverse momentum is presented. We have checked that the negative corrections for large transverse momenta are solely driven by the virtual contributions containing the Sudakov logarithms (not shown separately in the plots). The distribution in the missing transverse momentum in figure $6 \mathrm{~b}$ features the largest $\mathrm{EW}$ corrections which amount to $-8 \%$ at $400 \mathrm{GeV}$. The missing momentum is defined as the sum of the transverse momenta of the two neutrinos, i.e. $p_{\mathrm{T}, \mathrm{miss}}=\left|\mathbf{p}_{\mathrm{T}, \nu_{\mathrm{e}}}+\mathbf{p}_{\mathrm{T}, \bar{\nu}_{\mu}}\right|$. In figures $6 \mathrm{c}$ and $6 \mathrm{~d}$ the transverse momentum of the harder bottom quark (according to $p_{\mathrm{T}}$ ordering) and the transverse momentum of the bottomquark pair are displayed, respectively. Both observables receive corrections reaching $-6 \%$ at $400 \mathrm{GeV}$ and show similar behaviours. Figure $6 \mathrm{e}$ shows the distribution in the transverse momentum of the produced Higgs boson. At zero transverse momentum, the EW corrections amount to about $3 \%$ to reach $-6 \%$ for a Higgs boson with a transverse momentum of $400 \mathrm{GeV}$. Finally, the transverse momentum of the reconstructed top quark is displayed in figure $6 \mathrm{f}$. There, the $\mathrm{EW}$ corrections start at the level of $+4 \%$ below $50 \mathrm{GeV}$ to reach $-4 \%$ at $400 \mathrm{GeV}$.

In contrast to previous computations for top-quark-pair production [26, 68], the photon-induced contributions are stable over the whole range. The reason is that in the previous computations, the NNPDF23_nlo_as_0119_qed set [69-71] has been used, while for the present computation the LUXqed_plus_PDF4LHC15_nnlo_100 set [40] is employed. The latter features small photon PDF contributions and smaller errors. In particular, in the high-energy tail of distributions, the photon-induced contributions stay below one per cent while they were reaching $10 \%$ with previous PDF sets.

We turn to invariant-mass and angular distributions. As it is well known [19, 25, 26], the corrections to the reconstructed invariant top mass in figure 7 a display a radiative tail below the top-mass threshold. This is due to photons which have not been reconstructed with final-state particles. At $167 \mathrm{GeV}$, the corrections reach $+15 \%$ while at $181 \mathrm{GeV}$ they are practically zero. At the nominal top mass, the EW corrections amount to $-2 \%$.

The distribution in the invariant mass of the muon-antibottom system displayed in figure $7 \mathrm{~b}$ is particularly interesting. The threshold $M_{\mathrm{t}}^{2}-M_{\mathrm{W}}^{2} \simeq(154 \mathrm{GeV})^{2}$ marks the 
(a)
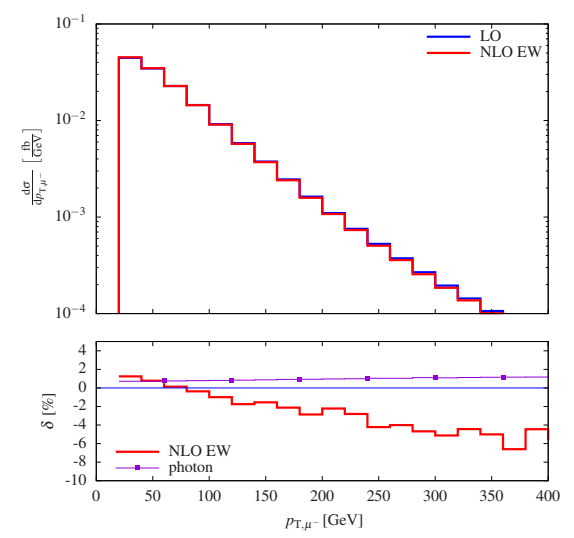

(c)
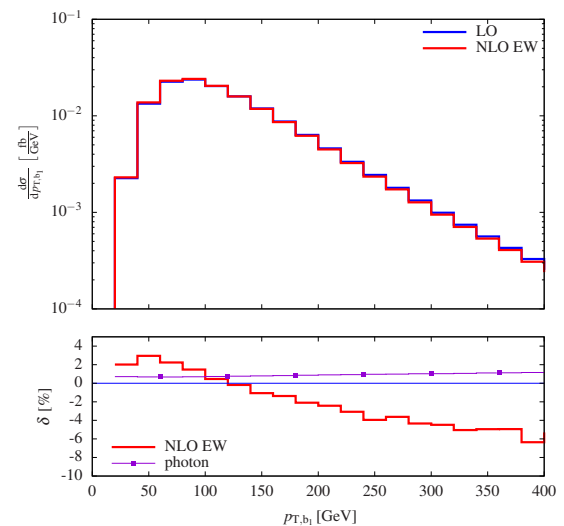

(e)
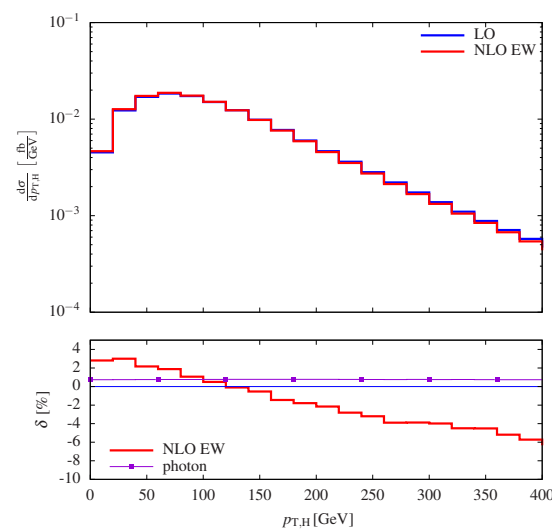

(b)
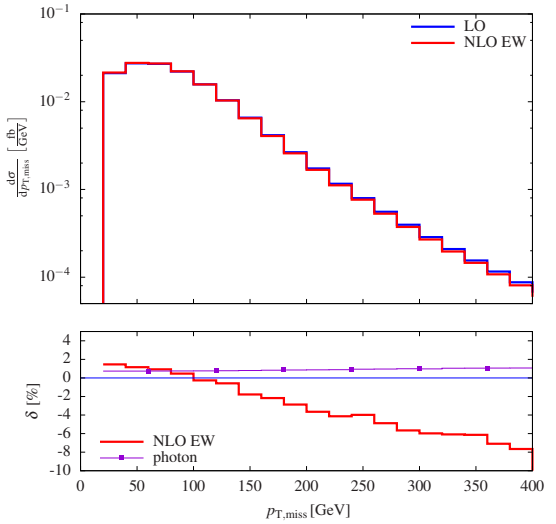

(d)
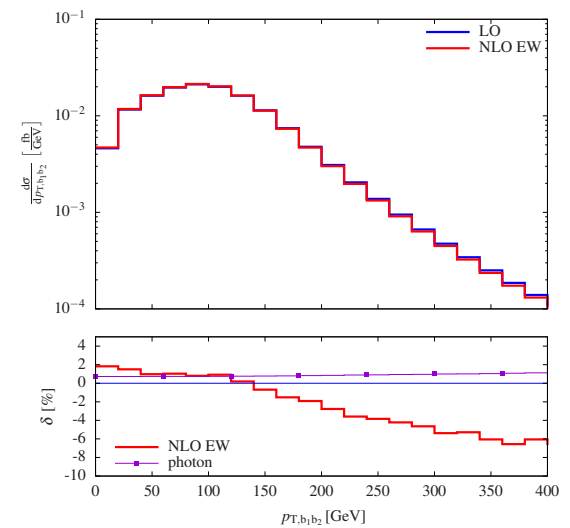

(f)

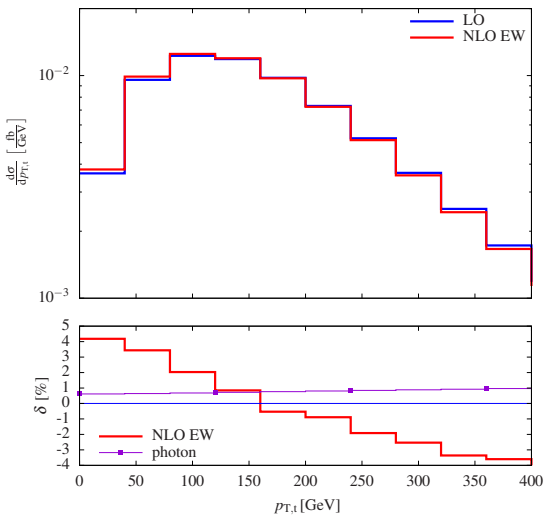

Figure 6. Transverse-momentum distributions at the LHC running at a centre-of-mass energy $\sqrt{s}=13 \mathrm{TeV}$ : (a) for the muon (upper left), (b) for the missing momentum (upper right), (c) for the harder bottom jet (middle left), (d) for the bottom-jet pair (middle right), (e) for the Higgs boson (lower left), and (f) for the reconstructed top quark (lower right). In the lower panels, the relative NLO EW corrections $\delta=\sigma_{\mathrm{NLO}} \mathrm{EW} / \sigma_{\mathrm{LO}}-1$ and the relative photon-induced contributions $\delta=\sigma_{\gamma g} / \sigma_{\mathrm{LO}}$ in per cent are shown. 
(a)

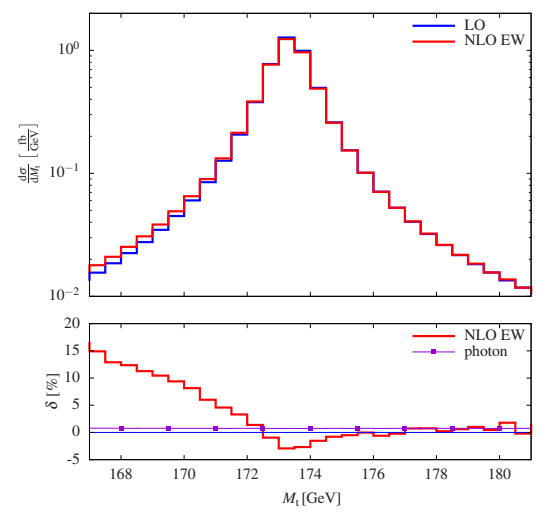

(c)
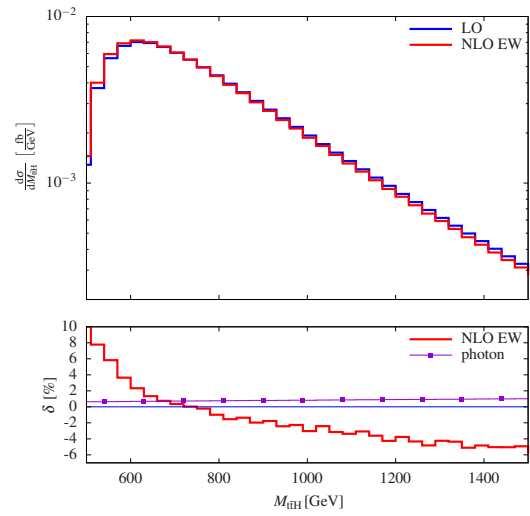

(e)

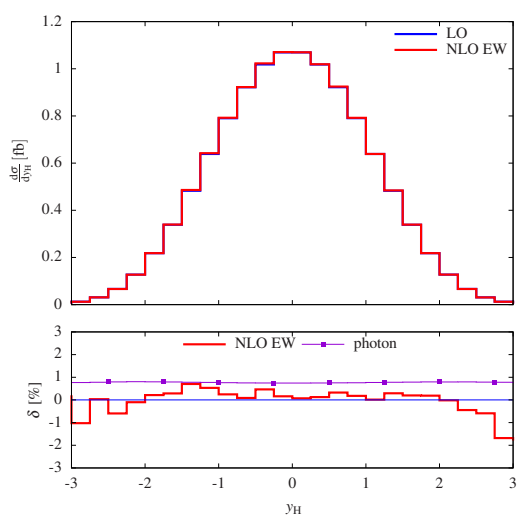

(b)

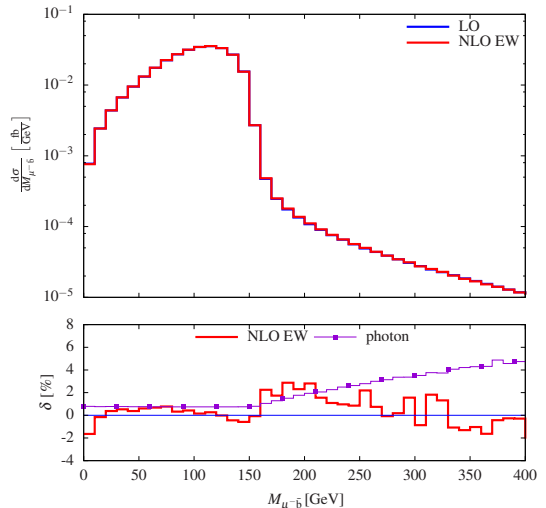

(d)

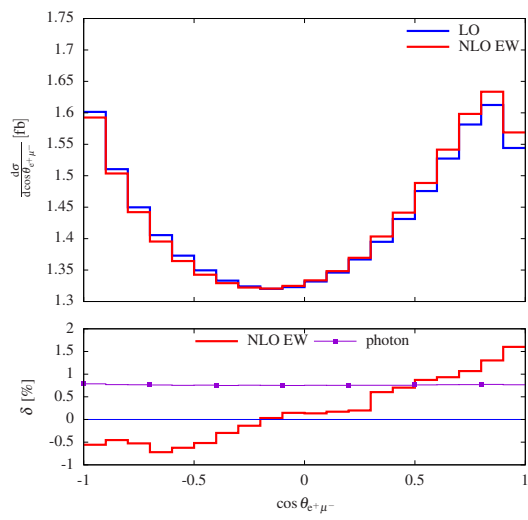

(f)

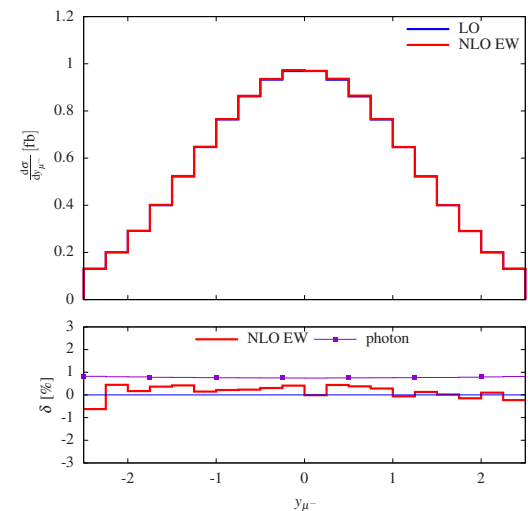

Figure 7. Distributions at the LHC running at a centre-of-mass energy $\sqrt{s}=13 \mathrm{TeV}$ : (a) invariant mass of the reconstructed top quark (upper left), (b) invariant mass of the $\mu^{-} \overline{\mathrm{b}}$ system (upper right), (c) invariant mass of the reconstructed t $\overline{\mathrm{t}} \mathrm{H}$ system (middle left), (d) cosine of the angle between the positron and the muon (middle right), (e) rapidity of the Higgs boson (lower left), and (f) rapidity of the muon (lower right). In the lower panels, the relative NLO EW corrections $\delta=\sigma_{\mathrm{NLO}} \mathrm{EW} / \sigma_{\mathrm{LO}}-1$ and the relative photon-induced contributions $\delta=\sigma_{\gamma g} / \sigma_{\mathrm{LO}}$ in per cent are shown. 
transition between the on-shell and off-shell production of the top-antitop quark pair [19, $25,26]$. Below and above this point, the EW corrections vary between $-1 \%$ and $+1 \%$ while they increase to about $+2 \%$ at threshold. One also notices that the photon-induced contributions are constant below threshold at the level of $1 \%$ but start to increase above threshold to reach $+5 \%$ at $400 \mathrm{GeV}$.

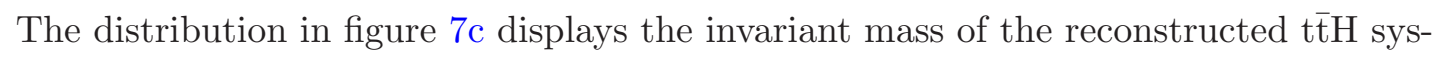
tem. It includes all the particles produced and thus shows the EW correction as a function of the partonic centre-of-mass energy. At $500 \mathrm{GeV}$, i.e. just above the threshold $2 m_{\mathrm{t}}+M_{\mathrm{H}} \simeq$ $472 \mathrm{GeV}$, the corrections are at the level of $10 \%$ and go down to $-5 \%$ at $1500 \mathrm{GeV}$.

The distribution in the cosine of the angle between the positron and the muon is displayed in figure $7 \mathrm{~d}$. As for QCD corrections (with a larger amplitude), the relative EW ones are increasing between $\cos \theta_{e^{+} \mu^{-}}=-1$ and $\cos \theta_{e^{+} \mu^{-}}=1$ from $-0.5 \%$ to $+1.5 \%$.

Finally, we show the rapidity distribution of both the Higgs boson and the muon in figures $7 \mathrm{e}$ and $7 \mathrm{f}$. The other rapidity distributions also do not display any particular EW corrections, and no variation is observed over the whole rapidity range.

For the observables involving the reconstructed top quarks and/or the final-state Higgs boson, qualitatively we have found similar results than the ones presented in ref. [22]. As for the total cross section, a quantitative comparison is not possible due to the event selection applied in our off-shell calculation.

\subsection{Comparison to the double-pole approximations}

In this section a study of two different DPAs for the off-shell production of top-quark pairs in association with a Higgs boson is presented. In particular, by comparing them to the full calculation at the level of integrated cross section and differential distributions, we can infer the quality of these two approximations. The first approximation requires two resonant top quarks, while for the second two resonant $\mathrm{W}$ bosons are demanded.

Integrated cross section. To start, we report results at LO for the integrated cross section for both the gg and $q \bar{q}$ channels in table 2 . While the WW DPA agrees with the full LO result within one per cent, the tt DPA only agrees at the level of $1.5-2 \%$. The WW DPA approximates the full calculation better as it comprises most diagrams of the full process. In particular, it contains all the doubly and singly top-quark resonant contributions as well as some non-resonant contributions while by definition the tt DPA only contains the doubly-resonant top-quark contributions. Nonetheless, the agreement matches the order of magnitude $\Gamma / M$ expected for a DPA. Since the $\gamma \mathrm{g}$ channels contribute below the per-cent level and the associated QCD corrections have been neglected, we have not studied the DPAs for the photon-induced channel.

For the NLO EW DPAs, only the two channels that have been computed in the DPAs are shown in table 3 . Since the $g q(/ \bar{q})$ interference channels do not have virtual corrections, we have not applied a DPA to them. Interestingly, both approximations reproduce the total cross section within a per mille. Such an observation has already been made in ref. [26] when computing the NLO EW corrections to the off-shell production of two top quarks. The reason is that the Born, real-subtracted part and the convolution operator ( $P$ and $K$ 


\begin{tabular}{|ccccc|}
\hline Ch. & $\sigma_{\mathrm{LO}}^{\mathrm{WW} D P A}[\mathrm{fb}]$ & $\delta_{\mathrm{LO}}^{\mathrm{WW} D P A}[\%]$ & $\sigma_{\mathrm{LO}}^{\mathrm{tt} D P A}[\mathrm{fb}]$ & $\delta_{\mathrm{LO}}^{\mathrm{tt} D P A}[\%]$ \\
\hline $\mathrm{gg}$ & $2.0003(1)$ & -0.56 & $1.9738(1)$ & -1.88 \\
$q \bar{q}$ & $0.8437(5)$ & -0.58 & $0.83640(5)$ & -1.44 \\
\hline $\mathrm{pp}$ & $2.8441(1)$ & -0.56 & $2.8102(1)$ & -1.75 \\
\hline
\end{tabular}

Table 2. Integrated LO cross sections for the two DPAs. The relative difference is defined as $\delta_{\mathrm{LO}}^{\mathrm{DPA}}=\sigma_{\mathrm{LO}}^{\mathrm{DPA}} / \sigma_{\mathrm{LO}}^{\text {Full }}-1$ in per cent.

\begin{tabular}{|ccccc|}
\hline Ch. & $\sigma_{\mathrm{NLO}}^{\mathrm{WW} \text { DPA }}[\mathrm{fb}]$ & $\delta_{\mathrm{NLO}}^{\mathrm{WW} \text { DPA }}[\%]$ & $\sigma_{\mathrm{NLO} \text { EW }}^{\mathrm{tt} \text { DPA }}[\mathrm{fb}]$ & $\delta_{\mathrm{NLO} \mathrm{EW}}^{\mathrm{tt} \text { DPA }}[\%]$ \\
\hline $\mathrm{gg}$ & $2.0237(6)$ & +0.18 & $2.0188(2)$ & -0.06 \\
$q \bar{q}$ & $0.8470(4)$ & +0.19 & $0.8446(4)$ & -0.09 \\
\hline $\mathrm{pp}$ & $2.8712(8)$ & +0.18 & $2.8639(4)$ & -0.07 \\
\hline
\end{tabular}

Table 3. Integrated NLO cross section for the two DPAs. Only the channels where the DPAs are applied are shown. The relative difference is defined as $\delta_{\mathrm{NLO}}^{\mathrm{DPA}}=\sigma_{\mathrm{NLO}}^{\mathrm{DPA}} / \sigma_{\mathrm{NLO}}^{\text {Full }}-1$ in per cent.

operator in ref. [37]) contributions have been computed with the full off-shell kinematics. The DPA is only applied to the virtual corrections and the $I$-operator which in our set-up amount to $-0.2 \%$ of the NLO predictions for the gg channel.

Differential distributions. In figure 8, the full calculation is compared with the two DPAs at the distribution level at both LO and NLO. In the upper panels, only the WW DPA at LO is displayed (as on a logarithmic scale the three other curves are indistinguishable). In the lower panels, the relative difference between the approximations and the full computation at both LO and NLO is shown. The deviation with respect to the full calculation is defined as $\delta=\sigma_{\mathrm{DPA}} / \sigma_{\text {Full }}-1$ and expressed in per cent. Hence, in the lower panel "LO tt" denotes $\sigma_{\mathrm{LO}}^{\text {tt DPA }} / \sigma_{\mathrm{LO}}^{\text {Full }}-1$, while "NLO WW" denotes $\sigma_{\mathrm{NLO}}^{\mathrm{WW}}$ DPA $/ \sigma_{\mathrm{NLO}}^{\text {Full }}-1$ for example. We recall that for the NLO prediction, the DPA is not applied to the LO contributions, the real corrections, and to the $P$ - and $K$-operator terms. The LO DPAs are, thus, not used in the computation of the NLO DPAs and are shown here only for reference.

The transverse momentum distributions of the Higgs boson (figure 8a), of the harder bottom jet (figure $8 \mathrm{~b}$ ), and of the $\mathrm{e}^{+} \mu^{-}$system (figure 8c) exhibit similar features at LO and NLO for both approximations. The WW DPA constitutes a better approximation than the tt one at LO and agrees with the full calculation within $1 \%$ over the full range. The LO tt DPA can deviate by up to $8 \%$ for the harder bottom jet at $400 \mathrm{GeV}$. On the other hand, at NLO, both approximations reproduce the full computation well. As for the total cross section, the reason is that the DPA is only applied to the subtracted virtual corrections. In addition, we only show distributions up to $400 \mathrm{GeV}$ where off-shell and non-resonant contributions are not sizeable yet. But for higher transverse momenta, as e.g. in ref. [26] for off-shell top-antitop production, larger deviations from the full calculation can be observed. For example, for the NLO tt DPA, the difference can reach $4 \%$ for the transverse momentum of the harder bottom jet at $800 \mathrm{GeV}$. The disagreement is less stringent here since the NLO EW corrections are smaller and thus the relative difference is also smaller. 
(a)
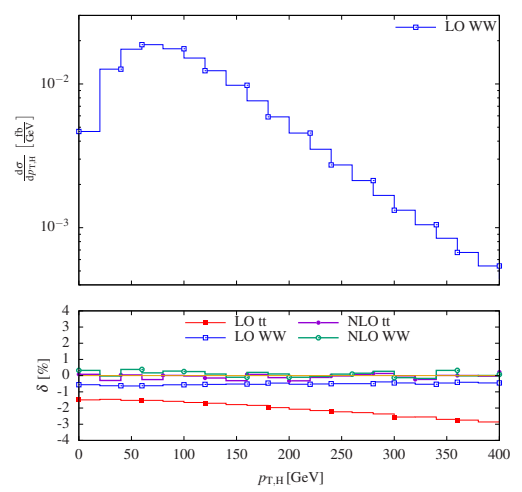

(c)

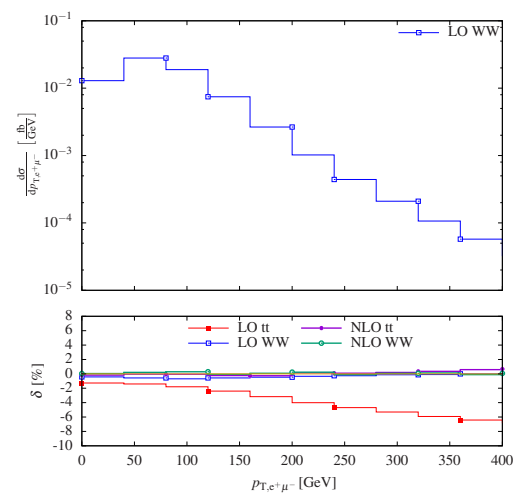

(e)

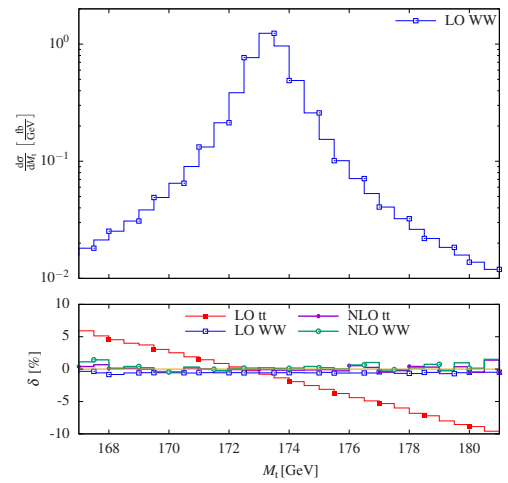

(b)

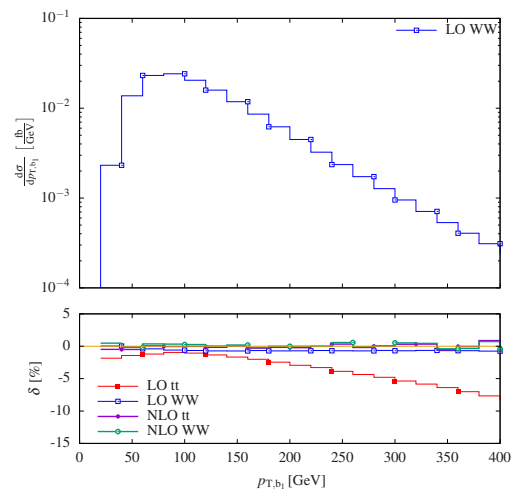

(d)

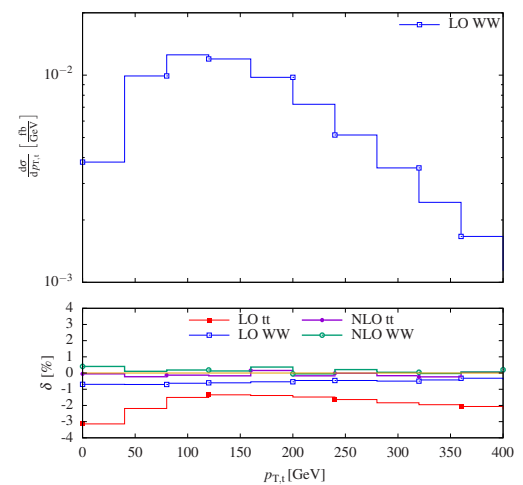

(f)

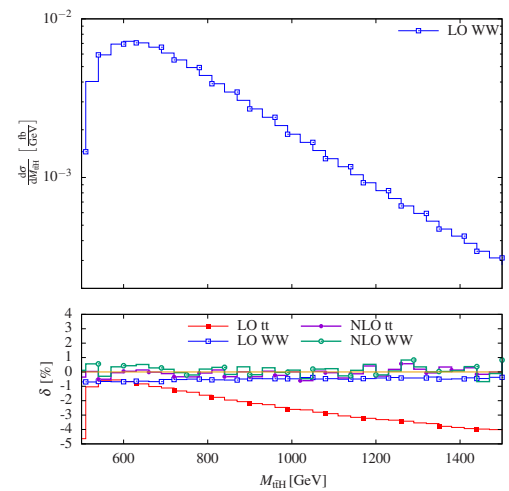

Figure 8. Comparison of the full calculation and the DPAs for various distributions at the LHC running at a centre-of-mass energy $\sqrt{s}=13 \mathrm{TeV}$ : (a) transverse momentum of the Higgs boson (upper left), (b) transverse momentum of the harder bottom jet (upper right), (c) transverse momentum of the $\mathrm{e}^{+} \mu^{-}$system (middle left) (d) transverse momentum of reconstructed top quark (middle right), (e) invariant mass of the reconstructed top quark (lower left), and (f) invariant mass of the reconstructed $t \bar{t} H$ system (lower right). In the upper panels the LO distributions for the WW DPA are shown. The lower panels display the relative deviation of the different DPAs from the full calculation, $\delta=\sigma_{\mathrm{DPA}} / \sigma_{\text {Full }}-1$, in per cent. 
The distribution in the transverse momentum of the reconstructed top quark is displayed in figure 8d. All approximations agree within one per cent apart from the LO tt DPA. The LO tt DPA is singled out from the other approximations because of its normalisation that disagrees by $1.75 \%$ with respect to the full LO. At zero transverse momentum, the disagreement is about $3 \%$ while it is $1.4 \%$ around $150 \mathrm{GeV}$ where the bulk of the distribution is located.

The invariant-mass distribution of the reconstructed top quark shown in figure $8 \mathrm{e}$ is interesting. All approximations agree with the full computation within $\pm 1 \%$ apart from the LO tt DPA. At $167 \mathrm{GeV}$, the LO tt DPA exceeds the full LO prediction by $5 \%$ while at $181 \mathrm{GeV}$ it is lower by $10 \%$. The full computation at LO and the LO tt DPA agree exactly at the top-quark mass value $(173.34 \mathrm{GeV})$.

The invariant mass of the reconstructed $t \bar{t} H$ system depicted in figure $8 \mathrm{f}$ shows similar features than the transverse-momentum distributions. The LO tt DPA tends to diverge from the full calculation towards higher invariant masses to disagree by almost $5 \%$ at $1500 \mathrm{GeV}$. The LO WW DPA on the other hand agrees perfectly over the full range. At NLO, both approximations describe the full computation well even at an invariant mass of $1500 \mathrm{GeV}$.

No rapidity distributions are displayed here as none of them shows any shape distortion between neither of the two DPAs and the full calculation. Differences for the LO tt DPA are only due to the different normalisation. For the distributions in the azimuthal-angle separation and the cosine of the angle between the two leptons, the shape distortions are also below one per cent.

To conclude, the tt DPA does not always provide a good description of the full calculation. This is particularly apparent in phase-space regions where off-shell and non-resonant contributions are sizeable. In these region, the DPA requiring two resonant top quarks can disagree by up to $4 \%$. On the contrary, for all inspected distributions, the WW DPA describes the full calculation within a per cent over the considered phase-space range.

\subsection{Combination of NLO EW and QCD corrections}

In this section, we present predictions for the integrated cross section as well as distributions taking into account both NLO EW and QCD corrections. These can be considered as state-of-the-art predictions for the production of a Higgs boson in association with a pair of off-shell top quarks. The NLO EW and QCD cross sections are defined as:

$$
\sigma_{\mathrm{QCD}}^{\mathrm{NLO}}=\sigma^{\mathrm{Born}}+\delta \sigma_{\mathrm{QCD}}^{\mathrm{NLO}} \quad \text { and } \quad \sigma_{\mathrm{EW}}^{\mathrm{NLO}}=\sigma^{\mathrm{Born}}+\delta \sigma_{\mathrm{EW}}^{\mathrm{NLO}},
$$

where the top width used in the top propagators includes both NLO QCD and EW corrections both at Born and NLO level. This allows a straight-forward additive combination of the two types of corrections:

$$
\sigma_{\mathrm{QCD}+\mathrm{EW}}^{\mathrm{NLO}}=\sigma^{\mathrm{Born}}+\delta \sigma_{\mathrm{QCD}}^{\mathrm{NLO}}+\delta \sigma_{\mathrm{EW}}^{\mathrm{NLO}}
$$

Based on the definition of eq. (3.7), the multiplicative combination can be defined as

$$
\sigma_{\mathrm{QCD} \times \mathrm{EW}}^{\mathrm{NLO}}=\sigma_{\mathrm{QCD}}^{\mathrm{NLO}}\left(1+\frac{\delta \sigma_{\mathrm{EW}}^{\mathrm{NLO}}}{\sigma^{\mathrm{Born}}}\right)=\sigma_{\mathrm{EW}}^{\mathrm{NLO}}\left(1+\frac{\delta \sigma_{\mathrm{QCD}}^{\mathrm{NLO}}}{\sigma^{\text {Born }}}\right) .
$$




\begin{tabular}{|cccccc|}
\hline$\sigma^{\mathrm{LO}}$ & $\sigma^{\text {Born }}$ & $\sigma_{\mathrm{QCD}}^{\mathrm{NLO}}$ & $\sigma_{\mathrm{EW}}^{\mathrm{NLO}}$ & $\sigma_{\mathrm{QCD}+\mathrm{EW}}^{\mathrm{NLO}}$ & $\sigma_{\mathrm{QCD} \times \mathrm{EW}}^{\mathrm{NLO}}$ \\
\hline $2.4817(1)$ & $2.7815(1)$ & $2.866(1)$ & $2.721(3)$ & 2.806 & 2.804 \\
\hline
\end{tabular}

Table 4. Integrated cross sections for $\mathrm{pp} \rightarrow \mathrm{e}^{+} \nu_{\mathrm{e}} \mu^{-} \bar{\nu}_{\mu} \mathrm{b} \overline{\mathrm{b}} \mathrm{H}(\gamma / \mathrm{j})$ at the LHC at a centre-of-mass energy of $\sqrt{s}=13 \mathrm{TeV}$. For $\sigma^{\mathrm{LO}}$, no NLO corrections are included in the top-quark width while for $\sigma^{\text {Born }}$ both NLO EW and QCD corrections are included. The latter Born contribution is the one used when computing the QCD and EW NLO predictions $\sigma_{\mathrm{QCD}}^{\mathrm{NLO}}$ and $\sigma_{\mathrm{EW}}^{\mathrm{NLO}}$, respectively. In addition, two ways of combining the NLO corrections are presented. They are defined in eqs. (3.8) and (3.9) for $\mathrm{QCD}+\mathrm{EW}$ and $\mathrm{QCD} \times \mathrm{EW}$, respectively. All cross sections are expressed in femtobarn $(\mathrm{fb})$.

The difference between these two ways of combining NLO EW and QCD corrections can give an estimate of the missing higher orders to the QCD-EW mixed contributions. The NLO QCD $\times$ EW combination can be understood as an improved prediction when the typical scales of the QCD and EW corrections are well separated. This is, for example, the case for soft QCD interactions with a scale well below the EW scale.

In table 4, a summary of the NLO QCD and EW corrections is presented. Note that in the present calculation $\sigma^{\text {Born }}$ and $\sigma^{\mathrm{LO}}$ are not identical. While $\sigma^{\mathrm{LO}}$ is computed with a top width without NLO corrections, $\sigma^{\text {Born }}$ is calculated with a top width featuring both NLO EW and QCD corrections. The latter Born contribution enters the cross sections $\sigma_{\mathrm{QCD}}^{\mathrm{NLO}}$ and $\sigma_{\mathrm{EW}}^{\mathrm{NLO}}$ at NLO QCD and EW, respectively. In addition the two possible ways of combining the NLO corrections are also given. The difference is negligible (of the order of the Monte Carlo error) and thus indicates that the missing higher orders to the QCD-EW mixed contributions are small.

We do not discuss the effects of the NLO QCD corrections as they have been investigated in detail in ref. [19], in particular, scale uncertainty and various differential distributions. We only discuss here results for the combination of both NLO EW and QCD corrections. Hence, in the upper panels the LO, NLO QCD+EW as well as the NLO QCD $\times$ EW predictions are displayed. In addition to predictions for the central scale we have also calculated predictions where both the renormalisation and factorisation scale are scaled by a factor 2 up and down. The envelope is obtained by taking the minimum and the maximum of these three predictions. In the lower panels the ratio of the LO and NLO predictions with respect to the LO prediction at the central scale is shown. Thus, the central curve for NLO corresponds to the usual $K$ factor $\mathrm{K}=\sigma_{\mathrm{NLO}} / \sigma_{\mathrm{LO}}$. The bands in the lower panels show the scale uncertainty resulting from the LO or NLO cross section in the numerator.

In general, the NLO effects are mainly driven by the QCD corrections as the EW corrections are smaller. For instance, this can be observed in the distribution of the bottom-jet pair shown in figure 9a. At $400 \mathrm{GeV}$ the combined $\mathrm{EW}$ and QCD corrections amount to about $74 \%$. There, the EW corrections reach $-6 \%$ while the $\mathrm{QCD}$ ones reach almost $80 \%$ [19]. Thus, even if the EW corrections are non-negligible, they look small by comparison to large QCD corrections. For this particular distribution, this huge effect is due to a kinematical constraint at $\mathrm{LO}$ which forces the transverse momenta of the bottom-jet-pair system to be strongly suppressed above $150 \mathrm{GeV}$. At NLO, this kinematical constraint is relaxed by the emission of an extra parton and thus leads to a huge $\mathrm{K}$ factor in this phase-space region. 
The difference between the $\mathrm{NLO}$ combinations $\mathrm{QCD}+\mathrm{EW}$ and $\mathrm{QCD} \times \mathrm{EW}$ gives an estimate of the missing higher orders of QCD-EW mixed contributions. In this regard, the two different ways of combining NLO effects give rather similar results. Consider for example the distribution in the missing transverse energy in figure $9 \mathrm{~b}$ where the $\mathrm{EW}$ corrections reach $8 \%$. At this very point, the two combinations differ by roughly two per cent at $400 \mathrm{GeV}$ indicating that missing higher orders are expected to be small in this phase-space region.

Figures 9c and 9d depict the transverse-momentum distribution of the Higgs boson and of the reconstructed top quark, respectively. These are key observables for a measurement of the Higgs production in association with top quarks.

Finally, the transverse momentum of the harder bottom jet and of the $\mathrm{e}^{+} \mu^{-}$system are shown in figures 9e and 9f, respectively. As the QCD corrections are positive, adding the EW corrections has the effect of damping the QCD corrections in the high-energy regime where Sudakov logarithms arise.

In figure 10 invariant-mass and angular distributions are presented. In figure 10a, a radiative tail resulting from non-recombined QCD-partons/photon is perfectly visible in the invariant-mass distribution of the reconstructed top quark. At $167 \mathrm{GeV}$, the effect of the EW corrections was found to be large (about $+15 \%$ as can be seen in figure $7 \mathrm{a}$ ). In the radiative tail $\mathrm{NLO} \mathrm{QCD}+\mathrm{EW}$ and $\mathrm{NLO} \mathrm{QCD} \times \mathrm{EW}$ differ by $50 \%$. Where $\mathrm{EW}$ (and $\mathrm{QCD}$ ) corrections are large, some higher-order contributions to mixed QCD-EW contributions might still be relevant.

The invariant mass of the reconstructed $t \bar{t} H$ system, displayed in figure 10b, shows the combined effect of the NLO QCD and EW correction as a function of the partonic centreof-mass energy. Both QCD and EW corrections diminish the cross section towards higher energies. In the distribution of the cosine of the angle between the positron and the muon (figure 10c), both NLO corrections give increasing contributions between $\cos \theta_{e^{+} \mu^{-}}=-1$ and $\cos \theta_{e^{+} \mu^{-}}=1$ ranging from $0 \%$ to $30 \%$. Finally, the distribution in the rapidity of the Higgs boson is shown in figure 10d. This prediction is basically dominated by the QCD effects as the EW corrections do not show any noticeable shape distortion over the whole range for this observable. This holds true as well for other rapidity distributions.

To conclude, the combined predictions at NLO QCD and EW are, as expected, mostly dominated by QCD effects. Nonetheless, in some phase-space regions EW corrections are non-negligible and should be taken into account in any precise analysis. The difference between the additive and multiplicative combination turns out to be small apart for some observables where EW corrections are large. In particular, it is always smaller than the scale uncertainty of the NLO QCD corrections.

\section{Conclusions}

The production of the Higgs boson in association with two top quarks will soon be measured at the LHC. In that respect precise predictions directly comparable with experiments are of prime importance. This means that decay products of the top quarks should be included, and event selections should be applied to them. In that way, one obtains realistic predictions for the full process $\mathrm{pp} \rightarrow \mathrm{e}^{+} \nu_{\mathrm{e}} \mu^{-} \bar{\nu}_{\mu} \mathrm{b} \overline{\mathrm{b}} \mathrm{H}$. 
(a)
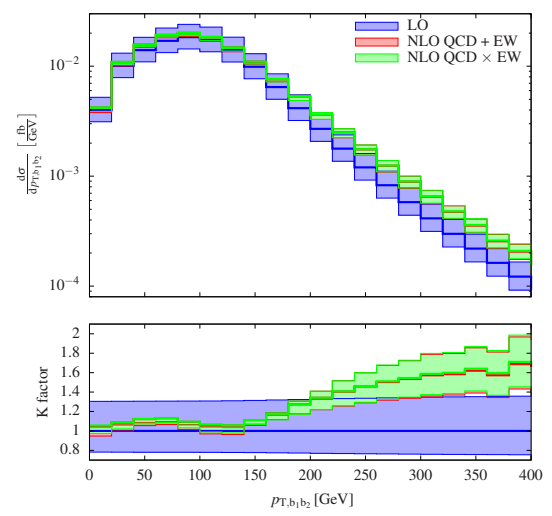

(c)
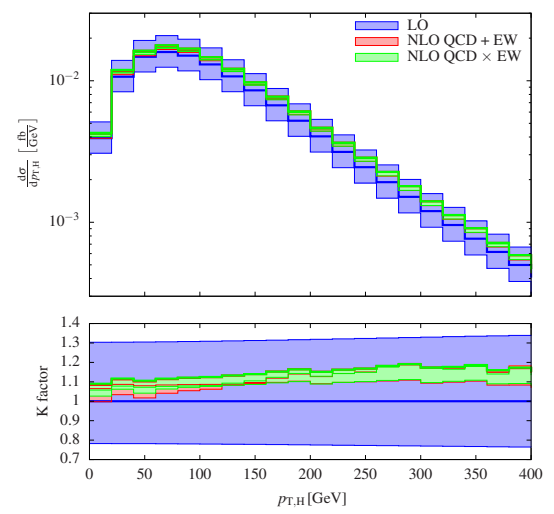

(e)
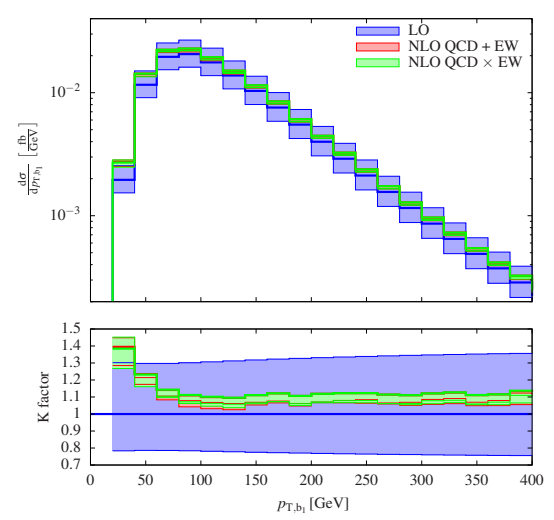

(b)
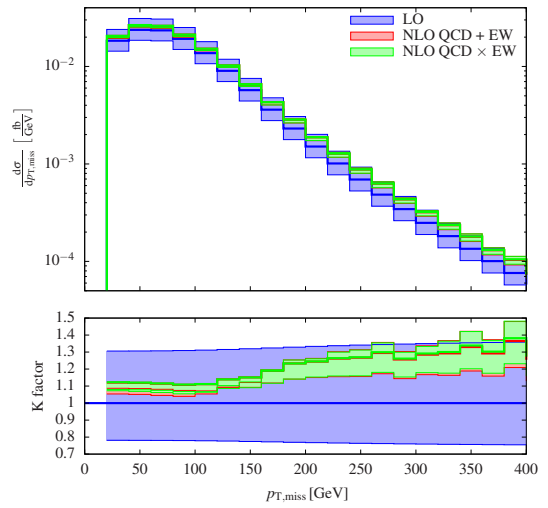

(d)

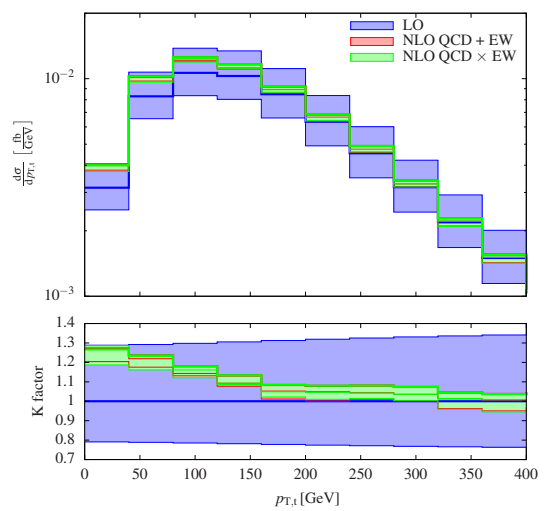

(f)

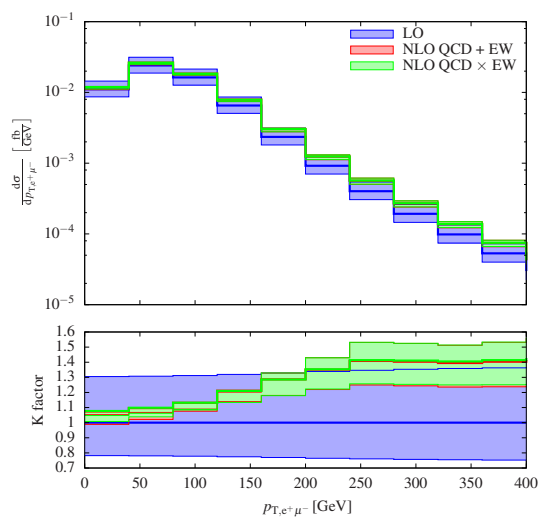

Figure 9. Combined NLO EW and QCD corrections for various distributions at the LHC running at a centre-of-mass energy $\sqrt{s}=13 \mathrm{TeV}$ : (a) transverse momentum for the bottom-jet pair (upper left), (b) for the missing momentum (upper right), (c) for the Higgs boson (middle left) (d) for the reconstructed top quark (middle right) (e) for the harder bottom jet (lower left), and (f) for the $\mathrm{e}^{+} \mu^{-}$system (lower right). In the upper panels the LO, the NLO QCD $+\mathrm{EW}$ and NLO QCD $\times \mathrm{EW}$ distributions are shown. The lower panels display the two differently combined NLO predictions with respect to the $\mathrm{LO}$ one as $\mathrm{K}=\sigma_{\mathrm{NLO}} / \sigma_{\mathrm{LO}}$. 
(a)
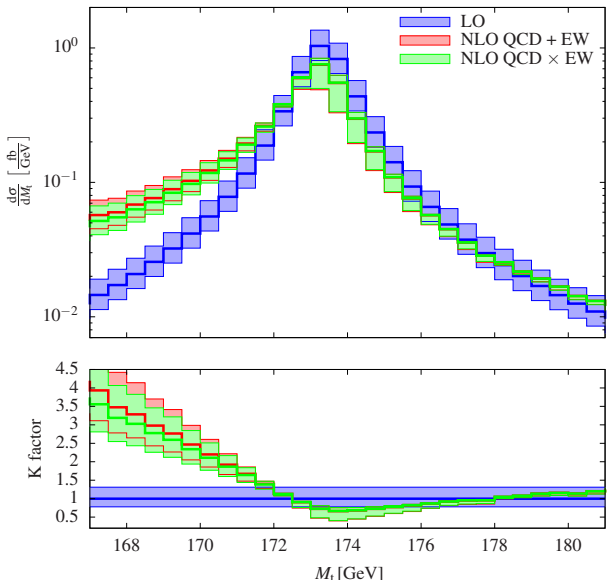

(c)

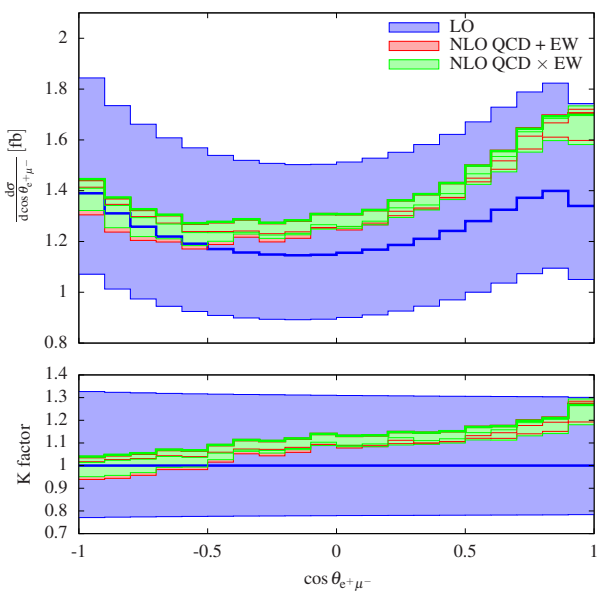

(b)
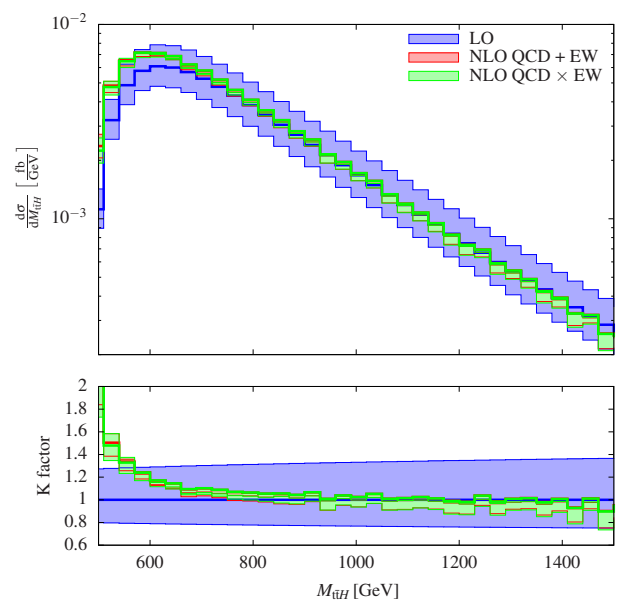

(d)
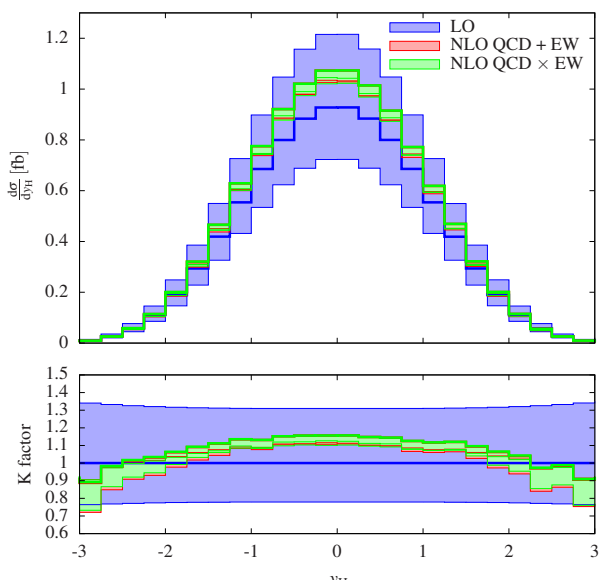

Figure 10. Combined NLO EW and QCD corrections for various distributions at the LHC running at a centre-of-mass energy $\sqrt{s}=13 \mathrm{TeV}$ : (a) invariant mass of the reconstructed top quark (upper left), (b) invariant mass of the reconstructed t $\mathrm{t} \mathrm{H}$ system (upper right), (c) cosine of the angle between the positron and the muon (lower left) (d) rapidity of Higgs boson (lower right). In the upper panels the LO, the NLO QCD+EW and NLO QCD $\times$ EW distributions are shown. The lower panels display the two combined NLO predictions with respect to the $\mathrm{LO}$ one as $\mathrm{K}=\sigma_{\mathrm{NLO}} / \sigma_{\mathrm{LO}}$.

For this process, the NLO QCD corrections have been recently computed, but the NLO electroweak (EW) ones were still missing. We have filled this gap by computing NLO EW corrections for the full process. This constitutes a particularly challenging computation especially for the virtual corrections. There, for the first time in a public NLO computation, one-loop amplitudes featuring up to 9-point functions appear. This is rendered possible by the use of the computer codes RECOLA and ColLien that can provide fast and reliable tree and one-loop amplitudes particularly suited for Monte Carlo simulations.

Our calculation of EW corrections to the full process pp $\rightarrow \mathrm{e}^{+} \nu_{\mathrm{e}} \mu^{-} \bar{\nu}_{\mu} \mathrm{b} \overline{\mathrm{b}} \mathrm{H}$ includes all interference, off-shell, and non-resonant contributions. The EW corrections to the 
fiducial cross section turn out to be small, i.e. below one per cent. Nonetheless, in certain phase-space regions, the EW corrections do become large. They can reach $-10 \%$ for some transverse-momentum distributions, e.g. the missing-transverse-energy distribution. The enhanced electroweak corrections are due to Sudakov logarithms that grow negatively large when all invariants are large. Other effects such as radiative tails resulting from real photon emission can also be sizeable. This occurs for example for the distribution in the invariant mass of the reconstructed top quarks where the EW corrections amount to $15 \%$. In the end, the inclusion of NLO EW corrections is mandatory in any precise analysis of Higgs production in association with two top quarks.

As the aforementioned computation is particularly challenging, we have supplemented it with two pole approximations. For the first approximation, two resonant top quarks are demanded, while in the second one two WW gauge bosons are required. Beyond checking the full computation, this also allows to investigate the impact of non-resonant contributions. Our results for the comparison are consistent with previous observations for the NLO EW corrections to the off-shell production of a top-antitop quark pair. When demanding only two resonant top quarks, errors with respect to the full calculation can reach $4 \%$ at $\mathrm{LO}$. On the other hand, requiring two $\mathrm{W}$ bosons seems to constitute a solid approximation. In all the distributions that we have computed, this approximation applied at LO has never been deviating by more than $2 \%$ with respect to the full calculation. At NLO, when using the double-pole approximation only for the subtracted virtual corrections, the differences to the full calculation stay below $1 \%$ for two resonant top quarks or two resonant $\mathrm{W}$ bosons. Nonetheless these conclusions only hold for the distributions that we have checked and for the shown phase-space range. In general one should rely on the full computation in order to ensure not to miss any off-shell or non-resonant effects.

In order to provide state-of-the-art predictions at NLO we have combined our NLO EW computation with an already existing NLO QCD computation. In this way, both effects are accounted for in a common and consistent set-up. Even if QCD effects are dominant, EW corrections are still important and should be taken into account. The additive and multiplicative combinations of the NLO corrections do not show large differences apart from some particular phase-space regions. This difference can be taken as an estimate of the missing higher-order contributions to mixed EW-QCD corrections.

The production of a Higgs boson in association with a pair of top quarks will probably soon be measured at the LHC. Thus, realistic and precise predictions will become very important. The present work will help the experimental collaborations to explore further the properties of the Higgs boson and maybe discover the existence of new physics.

\section{Acknowledgments}

We are grateful to Robert Feger for providing and supporting the code MoCANLO. The work of A.D. and M.P. was supported by the Bundesministerium für Bildung und Forschung (BMBF) under contract no. 05H15WWCA1 and the work of J.-N.L. by the Studienstiftung des Deutschen Volkes. The work of S.U. was supported in part by the European Commission through the "HiggsTools" Initial Training Network PITN-GA-2012-316704. 
Open Access. This article is distributed under the terms of the Creative Commons Attribution License (CC-BY 4.0), which permits any use, distribution and reproduction in any medium, provided the original author(s) and source are credited.

\section{References}

[1] ATLAS collaboration, Observation of a new particle in the search for the Standard Model Higgs boson with the ATLAS detector at the LHC, Phys. Lett. B 716 (2012) 1 [arXiv: 1207.7214] [INSPIRE].

[2] CMS collaboration, Observation of a new boson at a mass of $125 \mathrm{GeV}$ with the CMS experiment at the LHC, Phys. Lett. B 716 (2012) 30 [arXiv:1207.7235] [INSPIRE].

[3] ATLAS and CMS collaborations, Measurements of the Higgs boson production and decay rates and constraints on its couplings from a combined ATLAS and CMS analysis of the LHC pp collision data at $\sqrt{s}=7$ and $8 \mathrm{TeV}$, JHEP 08 (2016) 045 [arXiv:1606.02266] [INSPIRE].

[4] CMS collaboration, Search for the associated production of the Higgs boson with a top-quark pair, JHEP 09 (2014) 087 [Erratum ibid. 10 (2014) 106] [arXiv:1408.1682] [INSPIRE].

[5] ATLAS collaboration, Search for the associated production of the Higgs boson with a top quark pair in multilepton final states with the ATLAS detector, Phys. Lett. B $\mathbf{7 4 9}$ (2015) 519 [arXiv: 1506.05988] [INSPIRE].

[6] ATLAS collaboration, Search for the Standard Model Higgs boson produced in association with top quarks and decaying into $b \bar{b}$ in pp collisions at $\sqrt{s}=8 \mathrm{TeV}$ with the ATLAS detector, Eur. Phys. J. C 75 (2015) 349 [arXiv:1503.05066] [INSPIRE].

[7] ATLAS collaboration, Search for $H \rightarrow \gamma \gamma$ produced in association with top quarks and constraints on the Yukawa coupling between the top quark and the Higgs boson using data taken at $7 \mathrm{TeV}$ and $8 \mathrm{TeV}$ with the ATLAS detector, Phys. Lett. B 740 (2015) 222 [arXiv: 1409.3122] [INSPIRE].

[8] W. Beenakker, S. Dittmaier, M. Krämer, B. Plümper, M. Spira and P.M. Zerwas, Higgs radiation off top quarks at the Tevatron and the LHC, Phys. Rev. Lett. 87 (2001) 201805 [hep-ph/0107081] [INSPIRE].

[9] W. Beenakker, S. Dittmaier, M. Krämer, B. Plümper, M. Spira and P.M. Zerwas, NLO QCD corrections to $t \bar{t} H$ production in hadron collisions, Nucl. Phys. B 653 (2003) 151 [hep-ph/0211352] [INSPIRE].

[10] L. Reina and S. Dawson, Next-to-leading order results for tîth production at the Tevatron, Phys. Rev. Lett. 87 (2001) 201804 [hep-ph/0107101] [INSPIRE].

[11] S. Dawson, C. Jackson, L.H. Orr, L. Reina and D. Wackeroth, Associated Higgs production with top quarks at the Large Hadron Collider: NLO QCD corrections, Phys. Rev. D 68 (2003) 034022 [hep-ph/0305087] [INSPIRE].

[12] R. Frederix, S. Frixione, V. Hirschi, F. Maltoni, R. Pittau and P. Torrielli, Scalar and pseudoscalar Higgs production in association with a top-antitop pair, Phys. Lett. B 701 (2011) 427 [arXiv:1104.5613] [INSPIRE].

[13] M.V. Garzelli, A. Kardos, C.G. Papadopoulos and Z. Trócsányi, Standard Model Higgs boson production in association with a top anti-top pair at NLO with parton showering, Europhys. Lett. 96 (2011) 11001 [arXiv:1108.0387] [INSPIRE]. 
[14] H.B. Hartanto, B. Jäger, L. Reina and D. Wackeroth, Higgs boson production in association with top quarks in the POWHEG BOX, Phys. Rev. D 91 (2015) 094003 [arXiv:1501.04498] [INSPIRE].

[15] A. Kulesza, L. Motyka, T. Stebel and V. Theeuwes, Soft gluon resummation for associated $t \bar{t} H$ production at the LHC, JHEP 03 (2016) 065 [arXiv: 1509.02780] [INSPIRE].

[16] A. Broggio, A. Ferroglia, B.D. Pecjak, A. Signer and L.L. Yang, Associated production of a top pair and a Higgs boson beyond NLO, JHEP 03 (2016) 124 [arXiv:1510.01914] [INSPIRE].

[17] A. Kulesza, L. Motyka, T. Stebel and V. Theeuwes, Soft gluon resummation at fixed invariant mass for associated $t \bar{t} H$ production at the $L H C$, in $4^{\text {th }}$ Large Hadron Collider Physics Conference (LHCP 2016), Lund Sweden, 13-18 June 2016 [arXiv:1609.01619] [INSPIRE].

[18] A. Broggio, A. Ferroglia, B.D. Pecjak and L.L. Yang, NNLL resummation for the associated production of a top pair and a Higgs boson at the LHC, arXiv:1611.00049 [INSPIRE].

[19] A. Denner and R. Feger, NLO QCD corrections to off-shell top-antitop production with leptonic decays in association with a Higgs boson at the LHC, JHEP 11 (2015) 209 [arXiv: 1506.07448] [INSPIRE].

[20] B. Chokoufé Nejad, W. Kilian, J.M. Lindert, S. Pozzorini, J. Reuter and C. Weiss, NLO $Q C D$ predictions for off-shell $t \bar{t}$ and $t \bar{t} H$ production and decay at a linear collider, JHEP 12 (2016) 075 [arXiv: 1609.03390] [INSPIRE].

[21] S. Frixione, V. Hirschi, D. Pagani, H.S. Shao and M. Zaro, Weak corrections to Higgs hadroproduction in association with a top-quark pair, JHEP 09 (2014) 065 [arXiv: 1407.0823] [INSPIRE].

[22] S. Frixione, V. Hirschi, D. Pagani, H.S. Shao and M. Zaro, Electroweak and QCD corrections to top-pair hadroproduction in association with heavy bosons, JHEP 06 (2015) 184 [arXiv: 1504.03446] [INSPIRE].

[23] J.R. Andersen et al., Les Houches 2015: physics at TeV colliders Standard Model working group report, in $9^{\text {th }}$ Les Houches Workshop on Physics at TeV Colliders (PhysTeV 2015), Les Houches France, 1-19 June 2015 [arXiv: 1605.04692] [INSPIRE].

[24] Y. Zhang, W.-G. Ma, R.-Y. Zhang, C. Chen and L. Guo, QCD NLO and EW NLO corrections to $t \bar{t} H$ production with top quark decays at hadron collider, Phys. Lett. B 738 (2014) 1 [arXiv:1407.1110] [INSPIRE].

[25] A. Denner, S. Dittmaier, S. Kallweit and S. Pozzorini, NLO QCD corrections to off-shell top-antitop production with leptonic decays at hadron colliders, JHEP 10 (2012) 110 [arXiv:1207.5018] [INSPIRE].

[26] A. Denner and M. Pellen, NLO electroweak corrections to off-shell top-antitop production with leptonic decays at the LHC, JHEP 08 (2016) 155 [arXiv:1607.05571] [INSPIRE].

[27] Z. Bern et al., Next-to-leading order $W+5$-jet production at the LHC, Phys. Rev. D 88 (2013) 014025 [arXiv:1304.1253] [INSPIRE].

[28] B. Biedermann, A. Denner and M. Pellen, Large electroweak corrections to vector-boson scattering at the Large Hadron Collider, arXiv: 1611.02951 [INSPIRE].

[29] S. Actis, A. Denner, L. Hofer, A. Scharf and S. Uccirati, Recursive generation of one-loop amplitudes in the Standard Model, JHEP 04 (2013) 037 [arXiv:1211.6316] [INSPIRE]. 
[30] S. Actis, A. Denner, L. Hofer, J.-N. Lang, A. Scharf and S. Uccirati, RECOLA: REcursive Computation of One-Loop Amplitudes, Comput. Phys. Commun. (2017) in press [arXiv: 1605.01090] [INSPIRE].

[31] A. Denner, S. Dittmaier and L. Hofer, COLLIER - a fortran-library for one-loop integrals, PoS (LL2014) 071 [arXiv: 1407.0087] [INSPIRE].

[32] A. Denner, S. Dittmaier and L. Hofer, COLLIER: a fortran-based Complex One-Loop LIbrary in Extended Regularizations, Comput. Phys. Commun. 212 (2017) 220 [arXiv: 1604.06792] [INSPIRE].

[33] R. Feger, MoCaNLO: a generic Monte Carlo event generator for NLO calculations of hadron-collider processes, unpublished, (2015).

[34] F.A. Berends, R. Pittau and R. Kleiss, All electroweak four fermion processes in electron-positron collisions, Nucl. Phys. B 424 (1994) 308 [hep-ph/9404313] [INSPIRE].

[35] A. Denner, S. Dittmaier, M. Roth and D. Wackeroth, Predictions for all processes $e^{+} e^{-} \rightarrow 4$ fermions $+\gamma$, Nucl. Phys. B 560 (1999) 33 [hep-ph/9904472] [INSPIRE].

[36] S. Dittmaier and M. Roth, LUSIFER: a LUcid approach to SIx FERmion production, Nucl. Phys. B 642 (2002) 307 [hep-ph/0206070] [INSPIRE].

[37] S. Catani and M.H. Seymour, A general algorithm for calculating jet cross-sections in NLO QCD, Nucl. Phys. B 485 (1997) 291 [Erratum ibid. B 510 (1998) 503] [hep-ph/9605323] [INSPIRE].

[38] S. Catani, S. Dittmaier, M.H. Seymour and Z. Trócsányi, The dipole formalism for next-to-leading order QCD calculations with massive partons, Nucl. Phys. B 627 (2002) 189 [hep-ph/0201036] [INSPIRE].

[39] S. Dittmaier, A general approach to photon radiation off fermions, Nucl. Phys. B 565 (2000) 69 [hep-ph/9904440] [INSPIRE].

[40] A. Manohar, P. Nason, G.P. Salam and G. Zanderighi, How bright is the proton? A precise determination of the photon parton distribution function, Phys. Rev. Lett. 117 (2016) 242002 [arXiv: 1607.04266] [INSPIRE].

[41] G. 't Hooft and M.J.G. Veltman, Scalar one loop integrals, Nucl. Phys. B 153 (1979) 365 [INSPIRE].

[42] W. Beenakker and A. Denner, Infrared divergent scalar box integrals with applications in the electroweak Standard Model, Nucl. Phys. B 338 (1990) 349 [INSPIRE].

[43] S. Dittmaier, Separation of soft and collinear singularities from one loop $N$ point integrals, Nucl. Phys. B 675 (2003) 447 [hep-ph/0308246] [INSPIRE].

[44] A. Denner and S. Dittmaier, Scalar one-loop 4-point integrals, Nucl. Phys. B 844 (2011) 199 [arXiv: 1005.2076] [INSPIRE].

[45] G. Passarino and M.J.G. Veltman, One loop corrections for $e^{+} e^{-}$annihilation into $\mu^{+} \mu^{-}$in the Weinberg model, Nucl. Phys. B 160 (1979) 151 [inSPIRE].

[46] A. Denner and S. Dittmaier, Reduction of one loop tensor five point integrals, Nucl. Phys. B 658 (2003) 175 [hep-ph/0212259] [INSPIRE].

[47] A. Denner and S. Dittmaier, Reduction schemes for one-loop tensor integrals, Nucl. Phys. B 734 (2006) 62 [hep-ph/0509141] [INSPIRE]. 
[48] A. Denner, S. Dittmaier, M. Roth and L.H. Wieders, Electroweak corrections to charged-current $e^{+} e^{-} \rightarrow 4$ fermion processes: technical details and further results, Nucl. Phys. B 724 (2005) 247 [Erratum ibid. B 854 (2012) 504] [hep-ph/0505042] [INSPIRE].

[49] A. Denner and S. Dittmaier, The complex-mass scheme for perturbative calculations with unstable particles, Nucl. Phys. Proc. Suppl. 160 (2006) 22 [hep-ph/0605312] [INSPIRE].

[50] A. Denner, S. Dittmaier, M. Roth and D. Wackeroth, Electroweak radiative corrections to $e^{+} e^{-} \rightarrow W W \rightarrow 4$ fermions in double pole approximation: the RACOONWW approach, Nucl. Phys. B 587 (2000) 67 [hep-ph/0006307] [INSPIRE].

[51] E. Accomando, A. Denner and A. Kaiser, Logarithmic electroweak corrections to gauge-boson pair production at the LHC, Nucl. Phys. B 706 (2005) 325 [hep-ph/0409247] [INSPIRE].

[52] S. Dittmaier and C. Schwan, Non-factorizable photonic corrections to resonant production and decay of many unstable particles, Eur. Phys. J. C 76 (2016) 144 [arXiv:1511.01698] [INSPIRE].

[53] J. Alwall et al., The automated computation of tree-level and next-to-leading order differential cross sections and their matching to parton shower simulations, JHEP 07 (2014) 079 [arXiv:1405.0301] [INSPIRE].

[54] Z. Nagy and Z. Trócsányi, Next-to-leading order calculation of four jet observables in electron positron annihilation, Phys. Rev. D 59 (1999) 014020 [Erratum ibid. D 62 (2000) 099902] [hep-ph/9806317] [INSPIRE].

[55] J.R. Andersen et al., Les Houches 2013: physics at TeV colliders - Standard Model working group report, arXiv:1405.1067 [INSPIRE].

[56] A. Buckley et al., LHAPDF6: parton density access in the LHC precision era, Eur. Phys. J. C 75 (2015) 132 [arXiv:1412.7420] [INSPIRE].

[57] J. Butterworth et al., PDF4LHC recommendations for LHC run II, J. Phys. G 43 (2016) 023001 [arXiv: 1510.03865] [InSPIRE].

[58] S. Dulat et al., New parton distribution functions from a global analysis of quantum chromodynamics, Phys. Rev. D 93 (2016) 033006 [arXiv: 1506.07443] [INSPIRE].

[59] L.A. Harland-Lang, A.D. Martin, P. Motylinski and R.S. Thorne, Parton distributions in the LHC era: MMHT 2014 PDFs, Eur. Phys. J. C 75 (2015) 204 [arXiv:1412.3989] [InSPIRE].

[60] NNPDF collaboration, R.D. Ball et al., Parton distributions for the LHC run II, JHEP 04 (2015) 040 [arXiv: 1410.8849] [INSPIRE].

[61] J. Gao and P. Nadolsky, A meta-analysis of parton distribution functions, JHEP 07 (2014) 035 [arXiv: 1401.0013] [INSPIRE].

[62] S. Carrazza, S. Forte, Z. Kassabov, J.I. Latorre and J. Rojo, An unbiased Hessian representation for Monte Carlo PDFs, Eur. Phys. J. C 75 (2015) 369 [arXiv:1505.06736] [INSPIRE].

[63] Particle Data Group collaboration, J. Beringer et al., Review of particle physics (RPP), Phys. Rev. D 86 (2012) 010001 [INSPIRE].

[64] L. Basso, S. Dittmaier, A. Huss and L. Oggero, Techniques for the treatment of IR divergences in decay processes at NLO and application to the top-quark decay, Eur. Phys. J. C 76 (2016) 56 [arXiv:1507.04676] [INSPIRE]. 
[65] LhC Higgs Cross section Working Group collaboration, D. de Florian et al., Handbook of LHC Higgs cross sections: 4. Deciphering the nature of the Higgs sector, arXiv: 1610.07922 [INSPIRE].

[66] D. Yu. Bardin, A. Leike, T. Riemann and M. Sachwitz, Energy dependent width effects in $e^{+} e^{-}$annihilation near the $Z$ boson pole, Phys. Lett. B 206 (1988) 539 [INSPIRE].

[67] M. Cacciari, G.P. Salam and G. Soyez, The anti- $k_{t}$ jet clustering algorithm, JHEP 04 (2008) 063 [arXiv:0802.1189] [INSPIRE].

[68] D. Pagani, I. Tsinikos and M. Zaro, The impact of the photon PDF and electroweak corrections on $t \bar{t}$ distributions, Eur. Phys. J. C 76 (2016) 479 [arXiv:1606.01915] [INSPIRE].

[69] NNPDF collaboration, R.D. Ball et al., Parton distributions with QED corrections, Nucl. Phys. B 877 (2013) 290 [arXiv: 1308.0598] [InSPIRE].

[70] NNPDF collaboration, S. Carrazza, Towards the determination of the photon parton distribution function constrained by LHC data, PoS (DIS 2013) 279 [arXiv:1307.1131] [INSPIRE].

[71] NNPDF collaboration, S. Carrazza, Towards an unbiased determination of parton distributions with QED corrections, in Proceedings, $48^{\text {th }}$ Rencontres de Moriond on QCD and High Energy Interactions, La Thuile Italy, (2013), pg. 357 [arXiv: 1305.4179] [INSPIRE]. 